\title{
Preoperative Cardiopulmonary Exercise Test Associated with Postoperative Outcomes in Patients Undergoing Cancer Surgery: A Systematic Review and Meta-Analyses
}

\author{
Daniel Steffens, $\mathrm{PhD}^{1,2}$ (D) Hilmy Ismail, $\mathrm{MD}^{3,4}$, Linda Denehy, $\mathrm{PhD}^{3,4}$, Paula R. Beckenkamp, PhD $^{5}$, \\ Michael Solomon, DMed ${ }^{1,2}$, Cherry Koh, PhD ${ }^{1,2}$, Jenna Bartyn, MPH ${ }^{1}$, and Neil Pillinger, MB, BCh ${ }^{1,2}$ \\ ${ }^{1}$ Surgical Outcomes Research Centre (SOuRCe), Royal Prince Alfred Hospital (RPAH), Sydney, NSW, Australia; ${ }^{2}$ Faculty \\ of Medicine and Health, Central Clinical School, The University of Sydney, Sydney, Australia; ${ }^{3}$ School of Health \\ Sciences, The University of Melbourne, Melbourne, Australia; ${ }^{4}$ Peter MacCallum Cancer Centre, Melbourne, Australia; \\ ${ }^{5}$ Faculty of Health Sciences, Discipline of Physiotherapy, Musculoskeletal Health, The University of Sydney, Sydney, \\ Australia
}

\begin{abstract}
Backgrounds. There is mixed evidence on the value of preoperative cardiorespiratory exercise test (CPET) to predict postoperative outcomes in patients undergoing a cancer surgical procedure. The purpose of this review was to investigate the association between preoperative CPET variables and postoperative complications, length of hospital stay, and quality of life in patients undergoing cancer surgery.

Methods. A search was conducted on MEDLINE, Embase, AMED, and Web of science from inception to April 2020. Cohort studies investigating the association between preoperative CPET variables, including peak oxygen uptake (peak $\mathrm{VO}_{2}$ ), anaerobic threshold (AT), or ventilatory equivalent for carbon dioxide $\left(\mathrm{V}_{\mathrm{E}} / \mathrm{V}_{\mathrm{CO} 2}\right)$, and postoperative outcomes (complications, length of stay, and quality of life) were included. Risk of bias was assessed using the QUIPS tool. A random-effect model meta-analysis was performed whenever possible.
\end{abstract}

Supplementary Information The online version contains supplementary material available at https://doi.org/10.1245/s10434021-10251-3.

(C) Society of Surgical Oncology 2021

First Received: 18 March 2021

Accepted: 17 May 2021;

Published Online: 8 June 2021

D. Steffens, $\mathrm{PhD}$

e-mail: daniel.steffens@health.nsw.gov.au
Results. Fifty-two unique studies, including 10,030 patients were included. Overall, most studies were rated as having low risk of bias. Higher preoperative peak $\mathrm{VO}_{2}$ was associated with absence of postoperative complications (mean difference [MD]: 2.28; 95\% confidence interval [CI]: 1.26-3.29) and no pulmonary complication (MD: 1.47; 95\% CI: 0.49-2.45). Preoperative AT and $\mathrm{V}_{\mathrm{E}} / \mathrm{V}_{\mathrm{CO} 2}$ also demonstrated some positive trends. None of the included studies reported a negative trend.

Conclusions. This systematic review and meta-analysis demonstrated a significant association between superior preoperative CPET values, especially peak $\mathrm{VO}_{2}$, and better postoperative outcomes. The assessment of preoperative functional capacity in patients undergoing cancer surgery has the potential to facilitate treatment decision making.

The incidence of cancers in the global population is increasing. ${ }^{1}$ For selected patients, surgery with or without radiochemotherapy is the main treatment option. The goal of surgery is to obtain a clear resection margin and ultimately cure or prolong survival with an acceptable quality of life. ${ }^{2,3}$ However, despite the significant improvements in long-term survival over the recent years, the rate of postoperative morbidity remains high-increasing the length of hospital stay, reducing quality of life and contributing to a high treatment burden.

During the past 20 years, cardiopulmonary exercise test (CPET) was introduced during the preoperative period as an objective measure of functional capacity to evaluate the risk of adverse perioperative events and inform the 
perioperative management, particularly in high-risk patients undergoing high-risk surgery. ${ }^{4,5}$ Recently, CPET has gained popularity and is commonly used in high-risk patients undergoing cancer surgery in some surgical units. It is hypothesised that fitter patients, who were identified by using CPET, have greater physiological reserve to undergo surgery and recover sooner with fewer postoperative complications. ${ }^{6}$ This is extremely important for clinicians to inform decision-making, to better understand the postoperative course, and to guide postoperative management.

Several systematic reviews have explored the potential association between preoperative CPET variables and postoperative outcomes, demonstrating mixed results. While some systematic reviews have reported a significant positive association between preoperative CPET variables and postoperative complications, length of hospital stay, unplanned ICU admission, and 12-months survival, others have reported nonsignificant association. ${ }^{9-11}$ The prospective, multinational cohort (METS) study, for example, demonstrated an association between peak oxygen uptake (peak $\mathrm{VO}_{2}$ ) and noncardiac complications in a cohort of relatively well patients having noncardiac surgery and not limited to cancer surgery. ${ }^{12}$ Some limitations encountered within the previous systematic reviews include the absence of meta-analysis, inclusion of a mixed population (i.e., cancer and noncancer patients), outdated, or focused on a narrow cohort of patients. ${ }^{5,-11}$ Better understanding of the potential association between preoperative CPET variables and postoperative outcomes in cancer patients is extremely important; this can guide preoperative interventions designed to improve patients preoperative physical status. This, in turn, has the potential to reduce postoperative morbidity.

As the number of publications are rapidly growing further analysis, taking into consideration the limitations of the previous systematic reviews, is warranted. This systematic review aims to determine whether the preoperative CPET variables peak $\mathrm{VO}_{2}$, anaerobic threshold (AT), and ventilatory equivalent for carbon dioxide $\left(\mathrm{VE} / \mathrm{VCO}_{2}\right)$, are associated with postoperative complication rates, length of hospital stay, and quality of life in patients undergoing cancer surgery.

\section{METHODS}

\section{Protocol and Registration}

This systematic review was reported in accordance with the meta-analyses of Observational Studies in Epidemiology (MOOSE) checklist. ${ }^{13}$ The protocol for this systematic review was registered on the Open Science Framework website (https://osf.io/8ntvc/).
Information Sources and Search

A sensitive electronic search was performed via Ovid in MEDLINE, Embase, AMED, and Web of science via ww w.webofknowledge.com from inception to April 2020. An amalgamation of Medical Subject Headings (MeSH) terms and key words for "preoperative," "cardiopulmonary exercise test," and "neoplasm" was used in the search strategy (Supplementary Table 1). In addition, citation tracking of the included studies and relevant systematic reviews were conducted. The search was limited to humans with no date or language restrictions applied.

\section{Study Selection}

The screening process was conducted using Covidence (Covidence systematic review software, Veritas Health Innovation, Melbourne, Australia. Available at www.covi dence.org). The initial screening was completed by one review author removing clearly irrelevant studies (DS). Screening of titles and abstracts of potentially eligible studies was be performed by two independent review authors (DS and PRB) with full-text article assessed against the inclusion and exclusion criteria. Any disagreements over the eligibility of particular studies were resolved through discussion with a third review author (NP).

\section{Data Collection Process}

A standardized data form was used to extract data from eligible studies for assessment of the study quality and evidence synthesis. Two independent review authors extract the data independently (DS and PRB). Disagreements over the data extraction were resolved through discussion with a third reviewer (NP). The following information were extracted from each individual study: participant characteristics, study characteristics, CPET description and measures, postoperative outcome measures, and measures of association.

\section{Eligibility Criteria}

Longitudinal studies reporting on the association between preoperative CPET values and postoperative outcomes in adult patients aged $\geq 18$ years old undergoing a cancer-related surgical procedure were included if they reported the following: (i) at least one of the CPET measures of interest: (a) Peak Oxygen uptake (peak $\mathrm{VO}_{2}$ ): defined as the highest $\mathrm{VO}_{2}$ attained on a rapid incremental test. (b) Anaerobic threshold (AT): a submaximal index of exercise capacity defined as the oxygen uptake $\left(\mathrm{VO}_{2}\right)$ above which there is a metabolic transition to increased glycolysis and lactate begins to rise with an associated 
metabolic acidosis. (c) Ventilatory equivalent for carbon dioxide $\left(\mathrm{V}_{\mathrm{E}} / \mathrm{V}_{\mathrm{CO} 2}\right)$ : defined as the ratio of minute ventilation to carbon dioxide production usually reported at the AT; (ii) reported at least one postoperative outcome measure, including complication rate, length of hospital stay, and/or quality of life; (iii) Reported data on the association between preoperative CPET and postoperative outcome or provide enough data for the association to be calculated by the review authors.

Studies were excluded if they presented the following: (i) reported on mixed populations (e.g., cancer and noncancer patients, where the noncancer population $>5 \%$ of the investigated sample); (ii) the population of interest underwent open and close procedure (e.g., not completed as planned); (iii) abstracts of studies published on conference proceedings.

\section{Risk of Bias Assessment}

Risk of bias was assessed by using the Quality in Prognosis Studies (QUIPS) tool and was rated by two review authors (DS and PRB). ${ }^{14}$ Risk of bias was rated as "high", "moderate", or "low" risk according to the following domains: (i) Study participation; (ii) Study attrition; (iii) Outcome measurement; (vi) Statistical analysis and reporting. Due to the nature of this systematic review, the prognostic factor and study confounding domains were not judged as they were deemed not applicable. Disagreements over the risk of bias were resolved through discussion with a third review author (NP).

\section{Strategy for Data Synthesis}

For studies reporting on the association between preoperative CPET values and postoperative outcomes using continuous data, measures of central tendency (i.e., mean, median) and dispersion (i.e., standard deviation, 95\% confidence intervals [CI]) were extracted. However, for the studies reporting on dichotomous data, the number of patients presenting high/low CPET values and presence or absence of postoperative outcomes were extracted. Whenever possible, mean values and standard deviation were estimated using previously published formulas in order to pool data. ${ }^{15}$ When raw data were available, mean difference and 95\% CI (continuous) or odds ratios and 95\% CI (dichotomous) were calculated. For homogeneous studies (e.g., presenting comparable measures of CPET and postoperative outcomes) reporting on the association between preoperative CPET and postoperative outcomes a meta-analysis using a random-effect model was conducted. Studies presenting high variability of data types and format were presented descriptively. A post-hoc subgroup analysis was performed to investigate the association between preoperative CPET variables and postoperative outcomes according to cancer type. Pooled estimates were obtained with Comprehensive Meta-Analysis Software V.3 (Biostat, Englewood, NJ).

\section{RESULTS}

\section{Study Selection}

The electronic search yielded 843 potential studies after duplicates were removed. Of these, 212 full-text articles were considered for inclusion. A total of 58 published articles (including 52 unique cohorts) were included in this systematic review. The flow diagram of the inclusion process is presented in Fig. 1.

\section{Study Characteristics}

Of the 52 unique cohorts included, three included patients presenting with bladder cancer, ${ }^{16-18} 5$ colorectal, ${ }^{19-23} 5$ esophageal, ${ }^{24-29} 3$ liver, ${ }^{30-32} 27$ lung, ${ }^{33-60} 3$ pancreatic, ${ }^{61-63} 1$ rectal, $^{64}$ and 5 included mixed cancer populations. ${ }^{65-70}$ The sample size of the included studies ranged from 8 to $1684 .^{51,60}$ Most of the preoperative CPETs were performed by using a cycle ergometer. Peak $\mathrm{VO}_{2}$ was assessed in most studies (88\%), followed by AT (44\%). All included studies reported postoperative complication as an outcome, whereas quality of life was not reported in any of the included studies. The characteristics of the individual studies are presented in Table 1.

\section{Risk of Bias}

Overall, most studies were rated as having low risk of bias. Study participation was the domain with higher risk of bias (46\% rated as moderate/high risk of bias), whereas study attrition (10\% rated as moderate risk of bias), outcome measurement (10\% rated as moderate risk of bias), and statistical analysis and reporting (13\% rated as moderate risk of bias) were rated with lower risk of bias. The risk of bias assessment for each of the included study can be found in Table 2 .

\section{Association between Preoperative CPET Values and Postoperative Complications}

Peak $\mathrm{VO}_{2}$ The association between preoperative Peak $\mathrm{VO}_{2}$ and postoperative complications are presented in Fig. 2, Supplementary Fig. 1, and Tables 3 and 4. Our pooled analysis demonstrated that patients with no postoperative complication presented for surgery with a higher Peak $\mathrm{VO}_{2}$ (MD: 2.28; 95\% CI: $1.26-3.29 ; \mathrm{I}^{2}=9 \%$ ) 
FIG. 1 Included studies

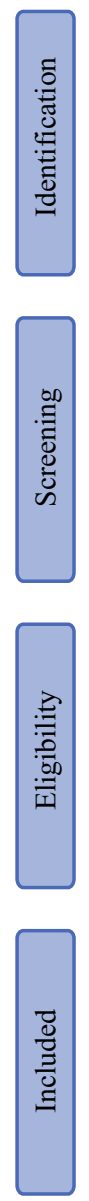

compared with patients who had postoperative complications (Fig. 2). A subgroup analysis in lung cancer patients demonstrated similar association (MD: 2.40; 95\% CI: 1.50-3.30) (Supplementary Fig. 2).

In addition, patients with no postoperative pulmonary complications (MD: 1.47; 95\% CI: 0.49-2.45; $\mathrm{I}^{2}=0 \%$ ), minor complications (MD: 2.01; 95\% CI: $0.90-3.13 ; I^{2}=$ 27\%), no cardiovascular complication (MD: 2.23; $95 \% \mathrm{CI}$ : 0.30-4.15), or no in-hospital mortality (MD: 2.78; 95\% CI: 1.12-4.43) compared with patients who presented with postoperative complications, presented for surgery with a significantly higher Peak $\mathrm{VO}_{2}$ (Fig. 2). No difference in Peak $\mathrm{VO}_{2}$ was found for patients with or without postoperative cardiopulmonary complications (Fig. 2). Other studies were not pooled in the meta-analysis due to high heterogeneity and reported mixed results (Tables 3 and 4).

$A T$ The association between preoperative AT and postoperative complications are presented in Fig. 3, Supplementary Fig. 1, and Tables 3 and 4. Our pooled analysis demonstrated no significant difference in preoperative AT values for patients with or without postoperative complications (MD: $0.15 ; 95 \% \mathrm{CI}:-0.32$ to 0.62 ) and cardiopulmonary complication (MD: 1.05; 95\% CI: -0.17 to $2.26 ; \mathrm{I}^{2}=0 \%$ ). Preoperative AT values were significantly higher in patients who presented minor complications compared with major complications (MD: 2.15; 95\% CI: $1.29-3.00 ; \mathrm{I}^{2}=0 \%$ ) and for no in-hospital mortality compared with in-hospital mortality (MD: 2.27; 95\% CI: 1.03-3.51) (Fig. 3). Other studies were not pooled in the meta-analysis due to heterogeneity and reported mixed results (Tables 3 and 4). Similar results were found on our subgroup analysis according to cancer type (Supplementary Fig. 3).

$V_{E} / V_{C O 2}$ The association between preoperative $\mathrm{V}_{\mathrm{E}} / \mathrm{V}_{\mathrm{CO} 2}$ and postoperative complications are presented in Fig. 4 and Tables 3 and 4. Our pooled analysis demonstrated that preoperative $\mathrm{V}_{\mathrm{E}} / \mathrm{V}_{\mathrm{CO} 2}$ values were significant lower in patients with no pulmonary complication compared with patients with pulmonary complication (MD: 3.54; 95\% CI: $\left.1.82-5.25 ; I^{2}=0 \%\right)$. No significant differences in 
TABLE 1 Characteristics of the included studies

\begin{tabular}{|c|c|c|c|c|c|c|}
\hline Author, year & $\begin{array}{l}\text { Type of } \\
\text { cancer }\end{array}$ & Characteristics & $\begin{array}{l}\text { CPET } \\
\text { assessment }\end{array}$ & CPET variables & $\begin{array}{l}\text { Postoperative } \\
\text { outcomes }\end{array}$ & Definition postoperative outcomes \\
\hline Lamb 2016 & Bladder & $\begin{array}{l}\text { Mean age (SD): } 65.0 \\
\quad(9.4) \\
\text { Sample size: } 111 \\
\text { Female }(\%): 30 \\
\quad(27 \%)\end{array}$ & Cycloergometer & $\begin{array}{l}\text { Peak } \mathrm{VO}_{2}(\mathrm{ml} / \\
\quad \mathrm{kg} / \mathrm{min}) \\
\mathrm{AT}(\mathrm{ml} / \mathrm{kg} / \mathrm{min}) \\
\mathrm{V}_{\mathrm{E}} / \mathrm{V}_{\mathrm{CO} 2}\end{array}$ & $\begin{array}{l}\text { Minor/major } \\
\text { complication } \\
\text { LOS }\end{array}$ & $\begin{array}{l}\text { Clavien-Dindo classification ( } \geq 3 \text { major } \\
\text { complication) } \\
\text { No. of days spent in hospital from the day } \\
\text { of operation until the day the patient left } \\
\text { the hospital }\end{array}$ \\
\hline Prentis 2013 & Bladder & $\begin{array}{l}\text { Mean age (SD): } 69.6 \\
\quad(6.5) \\
\text { Sample size: } 69 \\
\text { Female }(\%): 21 \\
\quad(30 \%)\end{array}$ & Cycloergometer & $\begin{array}{l}\text { Peak } \mathrm{VO}_{2}(\mathrm{ml} / \\
\quad \mathrm{kg} / \mathrm{min}) \\
\mathrm{AT}(\mathrm{ml} / \mathrm{kg} / \mathrm{min}) \\
\mathrm{V}_{\mathrm{E}} / \mathrm{V}_{\mathrm{CO} 2}\end{array}$ & Any complication & Clavien-Dindo classification \\
\hline $\begin{array}{l}\text { Tolchard } \\
2015\end{array}$ & Bladder & $\begin{array}{l}\text { Mean age (SD): } 70.2 \\
\quad(10.3) \\
\text { Sample size: } 105 \\
\text { Female }(\%): 17 \\
\quad(16 \%)\end{array}$ & Cycloergometer & $\begin{array}{l}\text { Peak } \mathrm{VO}_{2}(\mathrm{ml} / \\
\quad \mathrm{kg} / \mathrm{min}) \\
\mathrm{AT}(\mathrm{ml} / \mathrm{kg} / \mathrm{min}) \\
\mathrm{V}_{\mathrm{E}} / \mathrm{V}_{\mathrm{CO} 2}\end{array}$ & $\begin{array}{l}\text { Minor/major } \\
\text { complication }\end{array}$ & $\begin{array}{l}\text { Clavien-Dindo classification ( } \geq 2 \text { major } \\
\text { complication) }\end{array}$ \\
\hline Bowles 2008 & Colorectal & $\begin{array}{l}\text { Mean age (SD): NR } \\
\text { Sample size: } 121 \\
\text { Female }(\%): \text { NR }\end{array}$ & NR & AT $(\mathrm{ml} / \mathrm{kg} / \mathrm{min})$ & $\begin{array}{l}\text { Minor/major } \\
\text { complication } \\
\text { Mortality }\end{array}$ & $\begin{array}{l}\text { Clavien-Dindo classification ( } \geq 3 \text { major } \\
\text { complication) } \\
\text { Not specified }\end{array}$ \\
\hline Chan 2016 & Colorectal & $\begin{array}{l}\text { Mean age (SD): } 85.0 \\
\quad(10.4) \\
\text { Sample size: } 48 \\
\text { Female }(\%): 18 \\
\quad(38 \%)\end{array}$ & Cycloergometer & $\begin{array}{l}\text { Peak } \mathrm{VO}_{2}(\mathrm{ml} / \\
\mathrm{kg} / \mathrm{min}) \\
\text { AT }(\mathrm{ml} / \mathrm{kg} / \mathrm{min})\end{array}$ & $\begin{array}{l}\text { Any complication } \\
\text { Minor/major } \\
\text { complication }\end{array}$ & $\begin{array}{l}\text { Clavien-Dindo classification } \\
\text { Clavien-Dindo classification ( } \geq 3 \text { major } \\
\text { complication) }\end{array}$ \\
\hline Mann 2020 & Colorectal & $\begin{array}{l}\text { Mean age (SD): } 71.7 \\
\quad(8.8) \\
\text { Sample size: } 1214 \\
\text { Female }(\%): 501 \\
\quad(41.3 \%)\end{array}$ & NR & $\begin{array}{l}\mathrm{AT}(\mathrm{ml} / \mathrm{kg} / \mathrm{min}) \\
\mathrm{V}_{\mathrm{E}} / \mathrm{V}_{\mathrm{CO} 2}\end{array}$ & $\begin{array}{l}\text { Mortality } \\
\text { LOS }\end{array}$ & $\begin{array}{l}\text { Unplanned critical care use was defined as } \\
\text { any unexpected admission or re- } \\
\text { admission to high dependency unit } \\
\text { (HDU) or ICU from the general surgical } \\
\text { ward } \\
\text { Death within } 30 \text { days from surgery } \\
\text { No. of days spent in hospital from the day } \\
\text { of operation until the day the patient left } \\
\text { the hospital }\end{array}$ \\
\hline $\begin{array}{l}\text { McSorley } \\
2018\end{array}$ & Colorectal & $\begin{array}{l}\text { Mean age (SD): NR } \\
\text { Sample size: } 38 \\
\text { Female }(\%): 8 \\
\quad(21 \%)\end{array}$ & Cycloergometer & $\begin{array}{c}\text { Peak } \mathrm{VO}_{2}(\mathrm{ml} / \\
\mathrm{kg} / \mathrm{min})\end{array}$ & $\begin{array}{l}\text { Any complication } \\
\text { Minor/major } \\
\quad \text { complication } \\
\text { LOS }\end{array}$ & $\begin{array}{l}\text { Not specified } \\
\text { Not specified } \\
\text { Not specified }\end{array}$ \\
\hline $\begin{array}{l}\text { Nikolopoulos } \\
\quad 2015\end{array}$ & Colorectal & $\begin{array}{l}\text { Mean age (SD): } 59.3 \\
\quad(12.7) \\
\text { Sample size: } 69 \\
\text { Female }(\%): 34 \\
\quad(49 \%)\end{array}$ & Cycloergometer & AT $(\mathrm{ml} / \mathrm{kg} / \mathrm{min})$ & $\begin{array}{l}\text { Minor/major } \\
\text { complication }\end{array}$ & $\begin{array}{l}\text { Major complications included respiratory } \\
\text { failure, pneumonia with radiological } \\
\text { evidence, pulmonary embolism, } \\
\text { myocardial infarction verified by rise in } \\
\text { cardiac enzymes and ECG changes, } \\
\text { cardiac arrhythmias and congestive heart } \\
\text { failure requiring treatment, renal failure, } \\
\text { and sepsis }\end{array}$ \\
\hline Forshaw 2008 & Esophageal & $\begin{array}{l}\text { Mean age (SD): } 64.4 \\
\quad(8.5) \\
\text { Sample size: } 78 \\
\text { Female }(\%): 14 \\
\quad(18 \%)\end{array}$ & Cycloergometer & $\begin{array}{l}\text { Peak } \mathrm{VO}_{2}(\mathrm{ml} / \\
\mathrm{kg} / \mathrm{min}) \\
\text { AT }(\mathrm{ml} / \mathrm{kg} / \mathrm{min})\end{array}$ & $\begin{array}{l}\text { Cardiopulmonary } \\
\text { complication } \\
\text { Unplanned ITU } \\
\text { admission } \\
\text { LOS }\end{array}$ & $\begin{array}{l}\text { Common Terminology Criteria for Adverse } \\
\text { events } \\
\text { Unplanned reintubation and mechanical } \\
\text { ventilation } \\
\text { No. of days spent in hospital from the day } \\
\text { of operation until the day the patient left } \\
\text { the hospital }\end{array}$ \\
\hline
\end{tabular}


TABLE 1 continued

\begin{tabular}{|c|c|c|c|c|c|c|}
\hline Author, year & $\begin{array}{l}\text { Type of } \\
\text { cancer }\end{array}$ & Characteristics & $\begin{array}{l}\text { CPET } \\
\text { assessment }\end{array}$ & CPET variables & $\begin{array}{l}\text { Postoperative } \\
\text { outcomes }\end{array}$ & Definition postoperative outcomes \\
\hline \multirow[t]{3}{*}{ Lam 2019} & \multirow[t]{3}{*}{ Esophageal } & $\begin{array}{l}\text { Mean age }(\mathrm{SD}) \text { : } \\
66.9(9.2)\end{array}$ & \multirow[t]{3}{*}{ Cycloergometer } & $\begin{array}{l}\text { Peak } \mathrm{VO}_{2}(\mathrm{ml} / \\
\mathrm{kg} / \mathrm{min})\end{array}$ & Any complication & $\begin{array}{l}\text { Esophageal Complications Consensus } \\
\text { Group definitions }\end{array}$ \\
\hline & & Sample size: 206 & & \multirow[t]{2}{*}{$\mathrm{AT}(\mathrm{ml} / \mathrm{kg} / \mathrm{min})$} & \multirow{2}{*}{$\begin{array}{l}\text { Cardiopulmonary } \\
\text { complication }\end{array}$} & \multirow{2}{*}{$\begin{array}{l}\text { Esophageal Complications Consensus } \\
\text { Group definitions }\end{array}$} \\
\hline & & $\begin{array}{l}\text { Female }(\%): 48 \\
\qquad(23 \%)\end{array}$ & & & & \\
\hline \multirow[t]{3}{*}{$\begin{array}{l}\text { Nagamatsu } \\
2001 / 1994\end{array}$} & \multirow[t]{3}{*}{ Esophageal } & $\begin{array}{l}\text { Mean age (SD): } \\
59.0(9.0)\end{array}$ & \multirow[t]{3}{*}{ Cycloergometer } & $\begin{array}{l}\text { Peak VO2 }(\mathrm{ml} / \\
\left.\mathrm{min} / \mathrm{m}^{2}\right)\end{array}$ & \multirow[t]{3}{*}{$\begin{array}{l}\text { Cardiopulmonary } \\
\text { complication }\end{array}$} & \multirow{3}{*}{$\begin{array}{l}\text { More than } 10 \text { days of mechanical } \\
\text { ventilatory support, more than } 3 \text { days of } \\
\text { continuous therapy for a pulmonary } \\
\text { complication, or more than } 3 \text { days of } \\
\text { therapy for cardiac arrhythmias }\end{array}$} \\
\hline & & Sample size: 91 & & \multirow[t]{2}{*}{$\mathrm{AT}\left(\mathrm{ml} / \mathrm{min} / \mathrm{m}^{2}\right)$} & & \\
\hline & & $\begin{array}{l}\text { Female }(\%): 3 \\
\quad(3.3 \%)\end{array}$ & & & & \\
\hline \multirow[t]{3}{*}{ Patel 2019} & \multirow[t]{3}{*}{ Esophageal } & $\begin{array}{l}\text { Mean age (SD): } \\
\quad 64.6(9.0)\end{array}$ & \multirow[t]{3}{*}{ Cycloergometer } & $\begin{array}{l}\text { Peak } \mathrm{VO}_{2}(\mathrm{ml} / \\
\mathrm{kg} / \mathrm{min})\end{array}$ & $\begin{array}{l}\text { Minor/major } \\
\text { complication }\end{array}$ & $\begin{array}{l}\text { Clavien-Dindo classification ( } \geq 3 \text { major } \\
\text { complication) }\end{array}$ \\
\hline & & Sample size: 120 & & $\mathrm{AT}(\mathrm{ml} / \mathrm{kg} / \mathrm{min})$ & LOS & \multirow{2}{*}{$\begin{array}{l}\text { No. of days spent in hospital from the day } \\
\text { of operation until the day the patient left } \\
\text { the hospital }\end{array}$} \\
\hline & & $\begin{array}{l}\text { Female }(\%): 20 \\
\quad(17 \%)\end{array}$ & & $\mathrm{V}_{\mathrm{E}} / \mathrm{V}_{\mathrm{CO} 2}$ & & \\
\hline \multirow[t]{5}{*}{ Sinclair 2017} & \multirow[t]{5}{*}{ Esophageal } & $\begin{array}{l}\text { Mean age }(\mathrm{SD}) \text { : } \\
\quad 66.0(8.9)\end{array}$ & \multirow[t]{5}{*}{ Cycloergometer } & $\begin{array}{l}\text { Peak } \mathrm{VO}_{2}(\mathrm{ml} / \\
\mathrm{kg} / \mathrm{min})\end{array}$ & \multirow[t]{3}{*}{ Any complication } & \multirow{3}{*}{$\begin{array}{l}\text { Cardiovascular (acute coronary syndrome, } \\
\text { heart failure, problematic atrial } \\
\text { fibrillation); Respiratory (pneumonia, } \\
\text { pulmonary embolism, acute respiratory } \\
\text { distress syndrome); Gastro-intestinal } \\
\text { (anastomotic leak); and other } \\
\text { complications }\end{array}$} \\
\hline & & Sample size: 240 & & $\mathrm{AT}(\mathrm{ml} / \mathrm{kg} / \mathrm{min})$ & & \\
\hline & & \multirow[t]{3}{*}{$\begin{array}{l}\text { Female }(\%): 59 \\
\qquad(25 \%)\end{array}$} & & $\mathrm{V}_{\mathrm{E}} / \mathrm{V}_{\mathrm{CO} 2}$ & & \\
\hline & & & & & $\begin{array}{l}\text { Cardiopulmonary } \\
\text { complications }\end{array}$ & Not specified \\
\hline & & & & & LOS & $\begin{array}{l}\text { No. of days spent in hospital from the day } \\
\text { of operation until the day the patient left } \\
\text { the hospital }\end{array}$ \\
\hline \multirow[t]{4}{*}{ Dunne 2014} & \multirow[t]{4}{*}{ Liver } & \multirow{4}{*}{$\begin{array}{l}\text { Mean age (SD): } \\
\quad 69.6(8.2) \\
\text { Sample size: } 197 \\
\text { Female }(\%): 59 \\
\quad(30 \%)\end{array}$} & \multirow[t]{4}{*}{ Cycloergometer } & \multirow{4}{*}{$\begin{array}{l}\text { Peak } \mathrm{VO}_{2}(\mathrm{ml} / \\
\mathrm{kg} / \mathrm{min}) \\
\mathrm{V}_{\mathrm{E}} / \mathrm{V}_{\mathrm{CO} 2}\end{array}$} & Any complication & Clavien-Dindo classification. \\
\hline & & & & & Minor/major & Clavien-Dindo classification ( $\geq 3$ major \\
\hline & & & & & complication & complication) \\
\hline & & & & & LOS & $\begin{array}{l}\text { No. of days spent in hospital from the day } \\
\text { of operation until the day the patient left } \\
\text { the hospital }\end{array}$ \\
\hline \multirow{4}{*}{$\begin{array}{l}\text { Kasivisvanathan } \\
\quad 2015\end{array}$} & Liver & Mean age (SD): & Cycloergometer & Peak $\mathrm{VO}_{2}(\mathrm{ml} /$ & Any complication & POMS score $\geq 1$ on postoperative Day 3 \\
\hline & & $63.2(11.3)$ & & & LOS & No. of days spent in hospital from the day \\
\hline & & Sample size: 104 & & & & of operation until the day the patient left \\
\hline & & $\begin{array}{l}\text { Female }(\%): 44 \\
\quad(42 \%)\end{array}$ & & & & the hospital \\
\hline Ulyett 2017 & Liver & $\begin{array}{l}\text { Mean age }(\mathrm{SD}) \text { : } \\
\quad 68.0(12.7)\end{array}$ & Cycloergometer & $\begin{array}{l}\text { Peak } \mathrm{VO}_{2}(\mathrm{ml} / \\
\mathrm{kg} / \mathrm{min})\end{array}$ & $\begin{array}{l}\text { Minor/major } \\
\text { complication }\end{array}$ & $\begin{array}{l}\text { Clavien-Dindo classification ( } \geq 3 \text { major } \\
\text { complication) }\end{array}$ \\
\hline & & Sample size: 172 & & $\mathrm{~V}_{\mathrm{E}} / \mathrm{V}_{\mathrm{CO} 2}$ & & \\
\hline & & $\begin{array}{l}\text { Female }(\%): 53 \\
\quad(31 \%)\end{array}$ & & & & \\
\hline Bayram 2007 & Lung & Mean age (SD): & Cycloergometer & Peak $\mathrm{VO}_{2}(\mathrm{ml} /$ & Postoperative & Cardiopulmonary \\
\hline & & 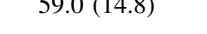 & & in) & comp & Pulmonary \\
\hline & & Sample size: 55 & & & & Respiratory \\
\hline & & Female (\%): 6 & & & & Mortality (30 days) \\
\hline & & & & & & Pneumonia \\
\hline & & & & & & Actelectasis \\
\hline & & & & & & Bronchopleural fistula \\
\hline & & & & & & Prolonged air leak \\
\hline & & & & & & Arrhytmia \\
\hline & & & & & LOS & $\begin{array}{l}\text { No. of days spent in hospital from the day } \\
\text { of operation until the day the patient left } \\
\text { the hospital }\end{array}$ \\
\hline
\end{tabular}


TABLE 1 continued

\begin{tabular}{|c|c|c|c|c|c|c|}
\hline Author, year & $\begin{array}{l}\text { Type of } \\
\text { cancer }\end{array}$ & Characteristics & $\begin{array}{l}\text { CPET } \\
\text { assessment }\end{array}$ & CPET variables & $\begin{array}{l}\text { Postoperative } \\
\text { outcomes }\end{array}$ & Definition postoperative outcomes \\
\hline $\begin{array}{l}\text { Bechard \& } \\
\text { Wetstein } 1987\end{array}$ & Lung & $\begin{array}{l}\text { Mean age (SD): } \\
\quad 63.8(6.5) \\
\text { Sample size: } 50 \\
\text { Female }(\%): 50 \\
\quad(100 \%)\end{array}$ & Cycloergometer & $\begin{array}{c}\text { Peak } \mathrm{VO}_{2}(\mathrm{ml} / \\
\mathrm{kg} / \mathrm{min}) \\
\mathrm{AT}(\mathrm{L} / \mathrm{min})\end{array}$ & Any complication & $\begin{array}{l}\text { Cardiopulmonary complications were } \\
\text { defined as acute } \mathrm{CO}_{2} \text { retention (partial } \\
\text { pressure of } \mathrm{CO}_{2}>45 \mathrm{~mm} \mathrm{Hg} \text { ), prolonged } \\
\text { mechanical ventilation ( }>48 \text { hours), } \\
\text { myocardial infarction, cardiac } \\
\text { arrhythmias necessitating therapy, } \\
\text { pneumonia (temperature }>38^{\circ} \mathrm{C} \text {, } \\
\text { purulent sputa, and infiltrate on chest } \\
\text { roentgenogram), pulmonary embolism } \\
\text { (high-probability ventilation/perfusion } \\
\text { scan or diagnostic pulmonary } \\
\text { angiogram), lobar atelectasis, and death }\end{array}$ \\
\hline Bobbio 2009 & Lung & $\begin{array}{l}\text { Mean age (SD): } \\
\quad 66.7(8.7) \\
\text { Sample size: } 73 \\
\text { Female }(\%): 12 \\
\quad(16 \%)\end{array}$ & Cycloergometer & $\begin{array}{c}\text { Peak } \mathrm{VO}_{2}(\mathrm{ml} / \\
\mathrm{kg} / \mathrm{min}) \\
\mathrm{V}_{\mathrm{E}} / \mathrm{V}_{\mathrm{CO} 2}\end{array}$ & $\begin{array}{l}\text { Pulmonary } \\
\text { complication }\end{array}$ & $\begin{array}{l}\text { Presence of pulmonary atelectasis requiring } \\
\text { bronchoscopy, in the case of pneumonia } \\
\text { (defined as a progressive radiological } \\
\text { infiltrate with fever and/or leukocytosis) } \\
\text { and in the case of respiratory failure }\end{array}$ \\
\hline Bolliger 1995 & Lung & $\begin{array}{l}\text { Mean age (SD): } \\
\quad 62.8(8.1) \\
\text { Sample size: } 25 \\
\text { Female }(\%): 8 \\
\quad(32 \%)\end{array}$ & Cycloergometer & $\begin{array}{c}\text { Peak } \mathrm{VO}_{2}(\mathrm{ml} / \\
\mathrm{kg} / \mathrm{min})\end{array}$ & Any complication & $\begin{array}{l}\text { Acute retention (partial pressure of arterial } \\
>45 \mathrm{~mm} \mathrm{Hg} \text { ); Prolonged mechanical } \\
\text { ventilation }(>48 \mathrm{~h} \text { ); Symptomatic } \\
\text { cardiac arrhythmias necessitating } \\
\text { treatment; Myocardial infarction; } \\
\text { Pneumonia (temperature }>38^{\circ} \mathrm{C} \text {, } \\
\text { purulent sputum, and infiltrate on chest } \\
\text { radiograph; Pulmonary embolism (high- } \\
\text { probability ventilation/perfusion scan or } \\
\text { diagnostic pulmonary angiogram); } \\
\text { Lobar atelectasis (necessitating } \\
\text { bronchoscopy); and Death }\end{array}$ \\
\hline Brat 2016 & Lung & $\begin{array}{l}\text { Mean age (SD): } \\
\quad 65.0(6.0) \\
\text { Sample size: } 76 \\
\text { Female }(\%): 27 \\
\quad(35 \%)\end{array}$ & Cycloergometer & $\begin{array}{c}\text { Peak } \mathrm{VO}_{2}(\mathrm{ml} / \\
\mathrm{kg} / \mathrm{min}) \\
\mathrm{V}_{\mathrm{E}} / \mathrm{V}_{\mathrm{CO} 2}\end{array}$ & $\begin{array}{l}\text { Pulmonary } \\
\text { complications }\end{array}$ & $\begin{array}{l}\text { Pneumonia (chest roentgenogram infiltrates } \\
\text { and at least two other markers including } \\
\text { fever or leukocytosis or leukopenia or } \\
\text { purulent sputum production); Atelectasis } \\
\text { (chest roentgenogram signs and } \\
\text { bronchoscopy with plug removal); } \\
\text { Respiratory failure requiring mechanical } \\
\text { ventilation (noninvasive ventilation or } \\
\text { tracheal intubation and invasive } \\
\text { pulmonary ventilation); Adult } \\
\text { respiratory distress syndrome (arterial } \\
\text { partial pressure of O O /fraction of } \\
\text { inspired } \mathrm{O}_{2}<300 \text { ); Pneumothorax } \\
\text { present on the third postoperative day, as } \\
\text { confirmed by chest roentgenogram } \\
\text { (changes or a new air-fluid level in case } \\
\text { of pneumonectomy), thoracic } \\
\text { ultrasound, or drain leak; tracheostomy. } \\
\text { Long-lasting pleural effusions present on } \\
\text { the third postoperative day, as confirmed } \\
\text { by chest roentgenogram (rapid filling of } \\
\text { the postpneumonectomy cavity with a } \\
\text { shift toward the opposite side in case of } \\
\text { pneumonectomy), thoracic ultrasound, } \\
\text { or drainage of more than } 200 \text { mL/day }\end{array}$ \\
\hline
\end{tabular}


TABLE 1 continued

\begin{tabular}{|c|c|c|c|c|c|c|}
\hline Author, year & $\begin{array}{l}\text { Type of } \\
\text { cancer }\end{array}$ & Characteristics & $\begin{array}{l}\text { CPET } \\
\text { assessment }\end{array}$ & CPET variables & $\begin{array}{l}\text { Postoperative } \\
\text { outcomes }\end{array}$ & Definition postoperative outcomes \\
\hline \multirow[t]{6}{*}{ Brunelli 2009} & \multirow[t]{6}{*}{ Lung } & \multirow{6}{*}{$\begin{array}{l}\text { Mean age (SD): } 66.5 \\
\quad(9.6) \\
\text { Sample size: } 204 \\
\text { Female }(\%): 35 \\
\quad(17 \%)\end{array}$} & \multirow[t]{6}{*}{ Cycloergometer } & \multirow{6}{*}{$\begin{array}{l}\text { Peak } \mathrm{VO}_{2}(\mathrm{ml} / \\
\mathrm{kg} / \mathrm{min}) \\
\text { AT }(\mathrm{ml} / \mathrm{kg} / \mathrm{min})\end{array}$} & Any complication & Any of the below defined complications \\
\hline & & & & & \multirow{3}{*}{$\begin{array}{l}\text { Pulmonary } \\
\text { complication }\end{array}$} & \multirow{3}{*}{$\begin{array}{l}\text { Respiratory failure: Assisted mechanical } \\
\text { ventilation for } 48 \text { h. Pneumonia: } \\
\text { Infiltrates seen on chest. ARDS: } \\
\text { Radiologic bilateral infiltrates. } \\
\text { Pulmonary edema: Radiologic and } \\
\text { clinical findings. Pulmonary embolism: } \\
\text { Confirmed by perfusion scan/CT scan }\end{array}$} \\
\hline & & & & & & \\
\hline & & & & & & \\
\hline & & & & & Cardiac complication & $\begin{array}{l}\text { Myocardial infarction: Suggestive ECG } \\
\text { findings and increased myocardial } \\
\text { enzymes; Arrhythmia: } \\
\text { Hemodynamically unstable and } \\
\text { requiring new treatment; Cardiac failure: } \\
\text { Suggestive radiograph findings, physical } \\
\text { examination findings, and symptoms; } \\
\text { Acute renal insufficiency: Change in } \\
\text { serum creatinine level } 2 \mathrm{mg} / \mathrm{dL} \\
\text { compared with preoperative values; } \\
\text { Stroke: Clinical findings/CT scan or } \\
\text { MRI }\end{array}$ \\
\hline & & & & & Mortality & In-hospital death \\
\hline \multirow[t]{3}{*}{ Brunelli 2012} & \multirow[t]{3}{*}{ Lung } & $\begin{array}{l}\text { Mean age (SD): } 67.2 \\
(9.8)\end{array}$ & \multirow[t]{3}{*}{ Cycloergometer } & $\begin{array}{l}\text { Peak } \mathrm{VO}_{2}(\mathrm{ml} / \\
\mathrm{kg} / \mathrm{min})\end{array}$ & \multirow[t]{3}{*}{$\begin{array}{l}\text { Pulmonary } \\
\text { complication }\end{array}$} & \multirow{3}{*}{$\begin{array}{l}\text { Pneumonia (chest roentgenogram } \\
\text { infiltrates/consolidation, leukocytosis, } \\
\text { fever), atelectasis requiring } \\
\text { bronchoscopy, respiratory failure } \\
\text { needing mechanical ventilation for }>48 \\
\text { hours, adult respiratory distress } \\
\text { syndrome (defined according to the } \\
\text { American-European consensus } \\
\text { conference), pulmonary edema, or } \\
\text { pulmonary embolism (confirmed by V/Q } \\
\text { scan or computed tomography scan) }\end{array}$} \\
\hline & & Sample size: 225 & & \multirow[t]{2}{*}{$\mathrm{V}_{\mathrm{E}} / \mathrm{V}_{\mathrm{CO} 2}$} & & \\
\hline & & $\begin{array}{l}\text { Female (\%): } 42 \\
\quad(19 \%)\end{array}$ & & & & \\
\hline \multirow[t]{3}{*}{$\begin{array}{l}\text { Brutsche } \\
2000\end{array}$} & \multirow[t]{3}{*}{ Lung } & $\begin{array}{l}\text { Mean age (SD): } 63.0 \\
\quad(11.0)\end{array}$ & \multirow[t]{3}{*}{ Cycloergometer } & \multirow[t]{3}{*}{$\begin{array}{l}\text { Peak } \mathrm{VO}_{2}(\mathrm{ml} / \\
\mathrm{kg} / \mathrm{min})\end{array}$} & \multirow[t]{3}{*}{ Any complication } & \multirow{3}{*}{$\begin{array}{l}\text { Acute carbon dioxide retention; Prolonged } \\
\text { mechanical ventilation }(>48 \mathrm{~h} \text { ); Treated } \\
\text { symptomatic cardiac arrhythmia; } \\
\text { Myocardial infarction; Pneumonia } \\
\text { (temperature }>38^{\circ} \mathrm{C} \text { and purulent } \\
\text { sputum and infiltrate on radiography); } \\
\text { Pulmonary embolism (high probability } \\
\text { on ventilation perfusion scan or } \\
\text { angiogram); Lobar atelectasis } \\
\text { (necessitating bronchoscopy); Death }\end{array}$} \\
\hline & & Sample size: 125 & & & & \\
\hline & & $\begin{array}{l}\text { Female (\%): } 24 \\
\quad(19 \%)\end{array}$ & & & & \\
\hline \multirow[t]{4}{*}{ Dales 1993} & \multirow[t]{4}{*}{ Lung } & \multirow{4}{*}{$\begin{array}{l}\text { Mean age (SD): NR } \\
\text { Sample size: } 46 \\
\text { Female (\%): NR }\end{array}$} & \multirow[t]{4}{*}{ Treadmill } & \multirow[t]{4}{*}{ Peak $\mathrm{VO}_{2}(\mathrm{~mL})$} & \multirow{3}{*}{$\begin{array}{l}\text { Respiratory } \\
\text { complications }\end{array}$} & \multirow[b]{3}{*}{$\begin{array}{l}\text { Atelectasis prompting bronchoscopy; } \\
\text { Pneumonia defined by a radiographic } \\
\text { infiltrate plus at least two of the } \\
\text { following: temperature }>37.7^{\circ} \mathrm{C} \text {, white } \\
\text { blood cell count }>10,500, \text { initiation of } \\
\text { antibiotics therapy, and demonstration of } \\
\text { pathogenic organisms; air leak or } \\
\text { effusion requiring intercostal tube } \\
\text { drainage }>7 \text { days; bronchopleural } \\
\text { fistula; empyema; chylothorax; } \\
\text { hemothoras requiring drainage or } \\
\text { reoperation; tension pneumothorax; } \\
\text { pulmonary embolism; lobar gangrene; } \\
\text { mechanical ventilation } \geq 72 \text { h for any } \\
\text { reason; intercostal tube drainage } \geq 14 \\
\text { days for any reason; and alveolar-arterial } \\
\text { oxygen gradient } \geq 300 \text { mm Hg } 24 \mathrm{~h} \\
\text { postoperatively }\end{array}$} \\
\hline & & & & & & \\
\hline & & & & & & \\
\hline & & & & & Any complication & $\begin{array}{l}\text { Included respiratory complication and } \\
\text { cardiac complications (myocardial } \\
\text { infarct defined by new-onset "Q" waves } \\
\text { or elevated CK-MB fraction, arrhythmia } \\
\text { requiring treatment, and congestive heart } \\
\text { failure defined by bilateral crackles, }\end{array}$ \\
\hline
\end{tabular}


TABLE 1 continued

\begin{tabular}{|c|c|c|c|c|c|c|}
\hline Author, year & $\begin{array}{l}\text { Type of } \\
\text { cancer }\end{array}$ & Characteristics & $\begin{array}{l}\text { CPET } \\
\quad \text { assessment }\end{array}$ & CPET variables & $\begin{array}{c}\text { Postoperative } \\
\text { outcomes }\end{array}$ & Definition postoperative outcomes \\
\hline
\end{tabular}

radiographic changes, or elevated
pulmonary artery wedge pressure and requiting therapy). Other complications were renal failure requiring dialysis, cerebrovascular accident, gastrointestinal bleeding, and wound infection

Epstein 1993 Lung
Mean age (SD): 62.6
(4.8)
Sample size: 42

Female (\%): $1(2 \%)$
Cycloergometer

$\mathrm{kg} / \mathrm{min})$
$\mathrm{Peak} \mathrm{VO}_{2}(\mathrm{~mL} /$
$\left.\mathrm{m}^{2}\right)$

Peak $\mathrm{VO}_{2}(\mathrm{~L})$
Any complication

Myocardial infarction (positive ECG

changes with elevated cardiac isoenzymes), unstable angina (appropriate clinical presentation with new ischemic ECG changes but normal isoenzyme levels), congestive heart failure (rales on physical examination with chest $x$-ray film showing pulmonary edema with pulmonary capillary wedge pressure, $\geq 18 \mathrm{~mm} \mathrm{Hg}$ or clinical response to diuretics), arrhythmia requiring therapy, reintubation, or prolonged mechanical ventilation ( $\geq 48 \mathrm{~h}$ after surgery), pneumonia (temperature $\geq 38^{\circ} \mathrm{C}$ for $\geq 48 \mathrm{~h}$ without an identifiable nonpulmonary source, plus purulent sputum and an infiltrate on the chest radiograph), lobar atelectasis requiring medical or bronchoscopic intervention, elevated [PaCO.sub.2] ( $\geq 50 \mathrm{~mm} \mathrm{Hg}$ or $\geq 10$-mm $\mathrm{Hg}$ increase from baseline lasting for $\geq 48 \mathrm{~h}$ after surgery), pulmonary embolism (high probability perfusion scan or abnormal pulmonary arteriogram), and death

\begin{tabular}{|c|c|c|c|c|}
\hline Fang 2014 & Lung & $\begin{array}{l}\text { Mean age (SD): } 67.3 \\
\quad(7.0) \\
\text { Sample size: } 107 \\
\text { Female (\%): } 3(2.8 \%)\end{array}$ & Cycloergometer & $\begin{array}{c}\text { Peak } \mathrm{VO}_{2}(\mathrm{ml} / \\
\mathrm{kg} / \mathrm{min})\end{array}$ \\
\hline Han 2007 & Lung & $\begin{array}{l}\text { Mean age (SD): } 65.0 \\
\quad(11.0) \\
\text { Sample size: } 467 \\
\text { Female (\%): } 184 \\
\quad(39 \%)\end{array}$ & NR & $\begin{array}{c}\text { Peak } \mathrm{VO}_{2}(\mathrm{ml} / \\
\mathrm{kg} / \mathrm{min})\end{array}$ \\
\hline $\begin{array}{l}\text { Kasikcioglu } \\
2009\end{array}$ & Lung & $\begin{array}{l}\text { Mean age (SD): } 61.0 \\
\quad(9.0) \\
\text { Sample size: } 49 \\
\text { Female }(\%): 5(10 \%)\end{array}$ & Treadmill & $\begin{array}{c}\text { Peak } \mathrm{VO}_{2}(\mathrm{ml} / \\
\mathrm{kg} / \mathrm{min})\end{array}$ \\
\hline Licker 2011 & Lung & $\begin{array}{l}\text { Mean age (SD): } 62.9 \\
\quad(10.7) \\
\text { Sample size: } 210 \\
\text { Female (\%): } 65 \\
\quad(31 \%)\end{array}$ & Cycloergometer & $\begin{array}{c}\text { Peak } \mathrm{VO}_{2}(\mathrm{ml} / \\
\mathrm{kg} / \mathrm{min})\end{array}$ \\
\hline
\end{tabular}

Any complication

Not specified
Pulmonary complication

Any complication
Atelectasis diagnosed by chest x-ray, pneumonia with sputum test, mechanical ventilation $>24$ h, reintubation, pulmonary embolism, ARDS, and pulmonary edema

Cardiopulmonary: prolonged mechanical ventilation $(>48 \mathrm{~h})$; respiratory insufficiency; lobar atelectasis on radiography; myocardial infarction verified by rise in enzymes; cardiac arrhythmias requiring therapy; pneumonia; heart failure requiring therapy; death caused by respiratory insufficiency or heart failure. Furthermore, technical related complications were defined as empyema; wound infections; leak of the bronchus stump; bronchopleural fistula; blood loss requiring transfusion
Any complication

Cardiovascular complication

Pulmonary complications
Any of the below defined complications

Myocardial infarction, arrhythmias, congestive heart failure, stroke, thromboembolism, or renal dysfunction

Atelectasis, pneumonia, or acute lung injury 
TABLE 1 continued

\begin{tabular}{|c|c|c|c|c|c|c|}
\hline Author, year & $\begin{array}{l}\text { Type of } \\
\text { cancer }\end{array}$ & Characteristics & $\begin{array}{l}\text { CPET } \\
\text { assessment }\end{array}$ & $\begin{array}{l}\text { CPET } \\
\text { variables }\end{array}$ & $\begin{array}{l}\text { Postoperative } \\
\text { outcomes }\end{array}$ & Definition postoperative outcomes \\
\hline Loewen 2007 & Lung & $\begin{array}{l}\text { Mean age (SD): } \\
\quad \text { NR } \\
\text { Sample size: } 403 \\
\text { Female (\%): } 158 \\
\quad(39 \%)\end{array}$ & Cycloergometer & $\begin{array}{l}\text { Peak } \mathrm{VO}_{2}(\mathrm{ml} / \\
\mathrm{kg} / \mathrm{min})\end{array}$ & $\begin{array}{l}\text { Cardiovascular } \\
\text { complication }\end{array}$ & $\begin{array}{l}\text { Red blood cell transfusion; Postoperative } \\
\text { fever; Wound infection; Empyema; } \\
\text { Prolonged air leak; Atelectasis } \\
\text { Pneumonia; Respiratory failure } \\
\text { Dysrhythmia; Myocardial infarction; } \\
\text { Deep vein thrombosis; Pulmonary } \\
\text { embolism; Postoperative death }\end{array}$ \\
\hline Mao 2010 & Lung & $\begin{array}{l}\text { Mean age (SD): } \\
\quad 64.7(11.5) \\
\text { Sample size: } 198 \\
\text { Female (\%): } 35 \\
\quad(18 \%)\end{array}$ & Cycloergometer & $\begin{array}{l}\text { Peak } \mathrm{VO}_{2}(\mathrm{ml} / \\
\mathrm{kg} / \mathrm{min})\end{array}$ & $\begin{array}{l}\text { Cardiopulmonary } \\
\text { complication }\end{array}$ & $\begin{array}{l}\text { Respiratory failure, pneumonitis/ } \\
\text { atelectasis, arrhythmia, supraventricular, } \\
\text { ventricular, myocardial infarction, heart } \\
\text { failure, severe shortness of breath, other } \\
\text { complications including pulmonary } \\
\text { artery embolism and gastrointestinal } \\
\text { tract bleeding }\end{array}$ \\
\hline Markos 1989 & Lung & $\begin{array}{l}\text { Mean age (SD): } \\
\quad 64.0(10.7) \\
\text { Sample size: } 47 \\
\text { Female }(\%): 17 \\
\quad(36 \%)\end{array}$ & Cycloergometer & $\begin{array}{l}\text { Peak } \mathrm{VO}_{2}(\mathrm{ml} / \\
\mathrm{kg} / \mathrm{min})\end{array}$ & Any complication & $\begin{array}{l}\text { Death, respiratory failure, pneumonia, lobar } \\
\text { atelectasis, pulmonary embolism, } \\
\text { myocardial infarction or ischemia, } \\
\text { symptomatic arrhythmias requiring } \\
\text { therapy, or admission to the intensive } \\
\text { care unit, or coronary care unit }\end{array}$ \\
\hline Miyazki 2018 & Lung & $\begin{array}{l}\text { Mean age (SD): } \\
\quad 72.4(8.3) \\
\text { Sample size: } 209 \\
\text { Female }(\%): 87 \\
\quad(42 \%)\end{array}$ & Cycloergometer & $\begin{array}{l}\text { Peak } \mathrm{VO}_{2}(\mathrm{ml} / \\
\mathrm{kg} / \mathrm{min}) \\
\mathrm{V}_{\mathrm{E}} / \mathrm{V}_{\mathrm{CO} 2}\end{array}$ & $\begin{array}{l}\text { Cardiopulmonary } \\
\text { complication }\end{array}$ & $\begin{array}{l}\text { Adult respiratory distress syndrome, } \\
\text { pneumonia, pulmonary embolism, } \\
\text { pulmonary edema, atelectasis requiring } \\
\text { bronchoscopy, respiratory failure ( }>24-\mathrm{h} \\
\text { mechanical ventilation or needing re- } \\
\text { intubation after surgery), arrhythmia } \\
\text { requiring electrical or medical } \\
\text { cardioversion, myocardial ischemia, } \\
\text { cardiac failure, stroke, and acute renal } \\
\text { failure }\end{array}$ \\
\hline Morice 1992 & Lung & $\begin{array}{l}\text { Mean age (SD): } 68 \\
\quad(3.8) \\
\text { Sample size: } 8 \\
\text { Female }(\%): 1 \\
\quad(13 \%)\end{array}$ & NR & $\begin{array}{l}\text { Peak } \mathrm{VO}_{2}(\mathrm{ml} / \\
\mathrm{kg} / \mathrm{min})\end{array}$ & $\begin{array}{l}\text { Cardiopulmonary } \\
\text { complication }\end{array}$ & $\begin{array}{l}\text { Mechanical ventilation }(>48 \mathrm{~h}) \text {; myocardial } \\
\text { infarction, as evidenced by EGG and } \\
\text { elevation of cardiac enzyme levels; } \\
\text { cardiac arrhythmias requiring short-term } \\
\text { therapy; pneumonia, defined as fever for } \\
48 \mathrm{~h} \text { and an infiltrate evident on chest } \\
\text { roentgenograms; roentgenographic } \\
\text { evidence of atelectasis; angiographically } \\
\text { documented pulmonary embolism; and } \\
\text { death within } 30 \text { days after surgery }\end{array}$ \\
\hline $\begin{array}{l}\text { Nagamatsu } 2004 \text { / } \\
\quad 2005\end{array}$ & Lung & $\begin{array}{l}\text { Mean age }(\mathrm{SD}): \\
\quad 65.9(8.4) \\
\text { Sample size: } 211 \\
\text { Female }(\%): 55 \\
\quad(26 \%)\end{array}$ & Cycloergometer & $\begin{array}{l}\text { Peak VO } 2(\mathrm{ml} / \\
\left.\mathrm{kg} / \mathrm{m}^{2}\right) \\
\text { AT }\left(\mathrm{ml} / \mathrm{kg} / \mathrm{m}^{2}\right)\end{array}$ & $\begin{array}{l}\text { Cardiopulmonary } \\
\text { complication }\end{array}$ & $\begin{array}{l}\text { Need for tracheostomy; mechanical } \\
\text { ventilation for at least } 2 \text { days; daily } \\
\text { bronchoscopic lavage for at least } 7 \text { days; } \\
\text { and the presence of arrhythmias } \\
\text { requiring treatment for at least } 3 \text { days }\end{array}$ \\
\hline Pate 1996 & Lung & $\begin{array}{l}\text { Mean age }(\mathrm{SD}) \text { : } \\
\quad 63.6(4.9) \\
\text { Sample size: } 12 \\
\text { Female }(\%): 2 \\
\quad(17 \%)\end{array}$ & Cycloergometer & $\begin{array}{c}\text { Peak } \mathrm{VO}_{2}(\mathrm{ml} / \\
\mathrm{kg} / \mathrm{min})\end{array}$ & Any complication & $\begin{array}{l}\text { Prolonged mechanical ventilation }(>48 \mathrm{~h}) \text {, } \\
\text { respiratory insufficiency (defined as } \\
\text { ventilator dependence or incapacitating } \\
\text { dyspnea as determined by survey), } \\
\text { persistent air leak ( }>10 \text { days), and } \\
\text { pneumonia; Arrhythmias, myocardial } \\
\text { infarction, pulmonary embolism, } \\
\text { hypotension, atelectasis, and death }\end{array}$ \\
\hline Rodrigues 2016 & Lung & $\begin{array}{l}\text { Mean age }(\mathrm{SD}) \text { : } \\
\quad 64.7(7.9) \\
\text { Sample size: } 50 \\
\text { Female }(\%): 4 \\
\quad(8 \%)\end{array}$ & Cycloergometer & $\begin{array}{l}\text { Peak } \mathrm{VO}_{2}(\mathrm{ml} / \\
\mathrm{kg} / \mathrm{min})\end{array}$ & $\begin{array}{l}\text { Pulmonary } \\
\text { complication }\end{array}$ & Not specified \\
\hline Torchio 2017 & Lung & $\begin{array}{l}\text { Mean age (SD): } 65 \\
\quad(8) \\
\text { Sample size: } 263 \\
\text { Female (\%): } 51 \\
\quad(19 \%)\end{array}$ & Treadmill & $\begin{array}{l}\text { Peak } \mathrm{VO}_{2}(\mathrm{ml} / \\
\mathrm{kg} / \mathrm{min}) \\
\mathrm{V}_{\mathrm{E}} / \mathrm{V}_{\mathrm{CO} 2}\end{array}$ & $\begin{array}{l}\text { Minor/major } \\
\text { complication }\end{array}$ & $\begin{array}{l}\text { Major complication defined if } \geq 1 \text { of the } \\
\text { following were present: cardiac failure } \\
\text { requiring inotropic support other than } \\
\text { renal dose dopamine; hemodynamically } \\
\text { unstable arrhythmia requiring treatment; } \\
\text { pulmonary embolism diagnosed by high- }\end{array}$ \\
\hline
\end{tabular}


TABLE 1 continued

\begin{tabular}{|c|c|c|c|c|c|c|}
\hline Author, year & $\begin{array}{l}\text { Type of } \\
\text { cancer }\end{array}$ & Characteristics & $\begin{array}{l}\text { CPET } \\
\text { assessment }\end{array}$ & $\begin{array}{l}\text { CPET } \\
\text { variables }\end{array}$ & $\begin{array}{c}\text { Postoperative } \\
\text { outcomes }\end{array}$ & Definition postoperative outcomes \\
\hline & & & & & & $\begin{array}{l}\text { probability perfusion scan or helical } \\
\text { computed tomographic scan; adult } \\
\text { respiratory distress syndrome; } \\
\text { respiratory failure }(\text { partial arterial } \\
\text { oxygen pressure }\left(\mathrm{PaO}_{2}\right)<65 \mathrm{~mm} \mathrm{Hg} \\
\text { and/or partial arterial carbon dioxide } \\
\left.\left.\text { pressure }(\mathrm{PaCO})_{2}\right) 45 \mathrm{~mm} \mathrm{Hg}\right) \text { requiring } \\
\text { noninvasive or invasive mechanical } \\
\text { ventilation; pneumonia defined by } \\
\text { typical clinical, laboratory, and } \\
\text { radiographic features; atelectasis } \\
\text { requiring bronchoscopy and/or } \\
\text { noninvasive assisted ventilation }\end{array}$ \\
\hline $\begin{array}{l}\text { Villani \& Busia } \\
2004\end{array}$ & Lung & $\begin{array}{l}\text { Mean age }(\mathrm{SE}) \text { : } \\
\quad 57.1(0.7) \\
\text { Sample size: } 150 \\
\text { Female }(\%): 9 \\
\quad(6 \%)\end{array}$ & Cycloergometer & $\begin{array}{l}\text { Peak } \mathrm{VO}_{2}(\mathrm{ml} / \\
\mathrm{kg} / \mathrm{min})\end{array}$ & Any complication & $\begin{array}{l}\text { Respiratory failure requiring oxygen } \\
\text { supplementation, lobar atelectasis, } \\
\text { cardiac arrhythmia requiring therapy, } \\
\text { pneumonia, acute respiratory distress } \\
\text { syndrome (ARDS), and pulmonary } \\
\text { embolism }\end{array}$ \\
\hline Win 2005 & Lung & $\begin{array}{l}\text { Mean age }(\mathrm{SD}) \text { : } \\
\quad 68.4(8.0) \\
\text { Sample size: } 99 \\
\text { Female }(\%): 38 \\
\quad(38 \%)\end{array}$ & Treadmill & $\begin{array}{l}\text { Peak } \mathrm{VO}_{2}(\mathrm{ml} / \\
\mathrm{kg} / \mathrm{min})\end{array}$ & Any complication & $\begin{array}{l}\text { Postoperative death, myocardial infarction, } \\
\text { heart failure, renal failure, respiratory } \\
\text { failure, pulmonary embolism, } \\
\text { septicemia, or pneumonia }\end{array}$ \\
\hline Yakal 2018 & Lung & $\begin{array}{l}\text { Mean age }(\mathrm{SD}) \text { : } \\
\quad 63.0(8.0) \\
\text { Sample size: } 125 \\
\text { Female }(\%): 19 \\
\quad(15 \%)\end{array}$ & Treadmill & $\begin{array}{l}\text { Peak } \mathrm{VO}_{2}(\mathrm{ml} / \\
\quad \mathrm{kg} / \mathrm{min}) \\
\mathrm{AT}(\mathrm{ml} / \mathrm{kg} / \\
\quad \mathrm{min}) \\
\mathrm{V}_{\mathrm{E}} / \mathrm{V}_{\mathrm{CO} 2}\end{array}$ & Any complication & Not specified \\
\hline Begum 2016 & Lung & $\begin{array}{l}\text { Mean age (SD): } \\
\quad \text { NR } \\
\text { Sample size: } 1684 \\
\text { Female }(\%) \text { : NR }\end{array}$ & NR & $\begin{array}{l}\text { Peak } \mathrm{VO}_{2}(\mathrm{ml} / \\
\mathrm{kg} / \mathrm{min})\end{array}$ & Cardiopulmonary & $\begin{array}{l}\text { Adult respiratory distress syndrome, } \\
\text { pneumonia, pulmonary embolism, } \\
\text { pulmonary edema, atelectasis requiring } \\
\text { bronchoscopy, respiratory failure, } \\
\text { arrhythmia requiring electrical or } \\
\text { medical cardioversion, myocardial } \\
\text { ischemia, cardiac failure, stroke, and } \\
\text { acute renal failure }\end{array}$ \\
\hline & & & & & Mortality & Death within 30 days from surgery \\
\hline Huang 2016 & Mixed & $\begin{array}{l}\text { Mean age (SD): } \\
\quad 67.7(9.6) \\
\text { Sample size: } 26 \\
\text { Female }(\%): 4 \\
\quad(15 \%)\end{array}$ & Cycloergometer & $\begin{array}{l}\text { Peak } \mathrm{VO}_{2}(\mathrm{ml} / \\
\mathrm{kg} / \mathrm{min})\end{array}$ & $\begin{array}{l}\text { Minor/major } \\
\text { complication }\end{array}$ & $\begin{array}{l}\text { Clavien-Dindo classification ( }>3 \text { major } \\
\text { complication) }\end{array}$ \\
\hline $\begin{array}{l}\text { Moyes } 2013 \\
\quad \text { Drummond } 2018\end{array}$ & Mixed & $\begin{array}{l}\text { Mean age }(\mathrm{SD}) \text { : } \\
\quad 66.0(9.0) \\
\text { Sample size: } 108 \\
\text { Female }(\%): 25 \\
\quad(23 \%)\end{array}$ & Cycloergometer & $\begin{array}{l}\text { Peak } \mathrm{VO}_{2}(\mathrm{ml} / \\
\mathrm{kg} / \mathrm{min}) \\
\mathrm{AT}(\mathrm{ml} / \mathrm{kg} / \\
\mathrm{min})\end{array}$ & $\begin{array}{l}\text { Cardiopulmonary } \\
\text { complication }\end{array}$ & $\begin{array}{l}\text { Common terminology criteria for adverse } \\
\text { events }\end{array}$ \\
\hline Snowden 2013 & Mixed & $\begin{array}{l}\text { Mean age }(\mathrm{SD}) \text { : } \\
\quad 65.8(10.3) \\
\text { Sample size: } 389 \\
\text { Female }(\%): 171 \\
\quad(44 \%)\end{array}$ & Cycloergometer & $\begin{array}{l}\text { Peak } \mathrm{VO}_{2}(\mathrm{ml} / \\
\quad \mathrm{kg} / \mathrm{min}) \\
\mathrm{AT}(\mathrm{ml} / \mathrm{kg} / \\
\quad \mathrm{min}) \\
\mathrm{V}_{\mathrm{E}} / \mathrm{V}_{\mathrm{CO} 2}\end{array}$ & Mortality & In-hospital death \\
\hline Whibley 2018 & Mixed & $\begin{array}{l}\text { Mean age }(\mathrm{SD}) \text { : } \\
\quad 64.9(9.5) \\
\text { Sample size: } 81 \\
\text { Female }(\%): \text { NR }\end{array}$ & NR & $\begin{array}{l}\text { Peak } \mathrm{VO}_{2}(\mathrm{ml} / \\
\mathrm{kg} / \mathrm{min}) \\
\mathrm{AT}(\mathrm{ml} / \mathrm{kg} / \\
\quad \mathrm{min})\end{array}$ & $\begin{array}{l}\text { Respiratory } \\
\text { complication }\end{array}$ & Not specified \\
\hline
\end{tabular}


TABLE 1 continued

\begin{tabular}{|c|c|c|c|c|c|c|}
\hline Author, year & $\begin{array}{l}\text { Type of } \\
\text { cancer }\end{array}$ & Characteristics & $\begin{array}{l}\text { CPET } \\
\text { assessment }\end{array}$ & CPET variables & $\begin{array}{l}\text { Postoperative } \\
\text { outcomes }\end{array}$ & Definition postoperative outcomes \\
\hline Wilson 2010 & Mixed & $\begin{array}{l}\text { Mean age (SD): } 72.2 \\
\quad(12.8) \\
\text { Sample size: } 847 \\
\text { Female (\%): } 341 \\
\quad(40 \%)\end{array}$ & Cycloergometer & $\begin{array}{l}\mathrm{AT}(\mathrm{ml} / \mathrm{kg} / \mathrm{min}) \\
\mathrm{V}_{\mathrm{E}} / \mathrm{V}_{\mathrm{CO} 2}\end{array}$ & $\begin{array}{l}\text { Mortality } \\
\text { LOS }\end{array}$ & $\begin{array}{l}\text { In-hospital death } \\
\text { No. of days spent in hospital from the day } \\
\text { of operation until the day the patient left } \\
\text { the hospital }\end{array}$ \\
\hline Ausania 2012 & Pancreas & $\begin{array}{l}\text { Mean age (SD): } 65.4 \\
\quad(8.7) \\
\text { Sample size: } 124 \\
\text { Female (\%): } 57 \\
\quad(46 \%)\end{array}$ & Cycloergometer & $\mathrm{AT}(\mathrm{ml} / \mathrm{kg} / \mathrm{min})$ & $\begin{array}{l}\text { Any complication } \\
\text { Mortality } \\
\text { LOS }\end{array}$ & $\begin{array}{l}\text { Postoperative morbidity survey } \\
\text { In-hospital mortality } \\
\text { No. of days spent in hospital from the day } \\
\text { of operation until the day the patient left } \\
\text { the hospital }\end{array}$ \\
\hline \multirow[t]{2}{*}{$\begin{array}{l}\text { Chandrabalan } \\
2013\end{array}$} & Pancreas & $\begin{array}{l}\text { Median age: } 66.0 \\
\text { Sample size: } 100 \\
\text { Female (\%): } 40 \\
\quad(40 \%)\end{array}$ & Cycloergometer & $\mathrm{AT}(\mathrm{ml} / \mathrm{kg} / \mathrm{min})$ & $\begin{array}{l}\text { Postoperative } \\
\text { complication }\end{array}$ & $\begin{array}{l}\text { The International Study Group for } \\
\text { Pancreatic Surgery (ISGPS) definitions } \\
\text { were used to classify pancreatic fistulae } \\
\text { and post-operative haemorrhage. The } \\
\text { Clavien-Dindo classification was used to } \\
\text { grade other complications ( } \geq 3 \text { major } \\
\text { complication) }\end{array}$ \\
\hline & & & & & LOS & $\begin{array}{l}\text { No. of days spent in hospital from the day } \\
\text { of operation until the day the patient left } \\
\text { the hospital }\end{array}$ \\
\hline \multirow[t]{3}{*}{ Junejo 2014} & Pancreas & $\begin{array}{l}\text { Mean age (SD): } 63.8 \\
\quad(7.5)\end{array}$ & Cycloergometer & $\begin{array}{l}\text { Peak } \mathrm{VO}_{2}(\mathrm{ml} / \\
\mathrm{kg} / \mathrm{min})\end{array}$ & Any complication & $\begin{array}{l}\text { International Study Group for Pancreatic } \\
\text { Surgery }\end{array}$ \\
\hline & & $\begin{array}{l}\text { Sample size: } 64 \\
\text { Female }(\%): 26 \\
\quad(41 \%)\end{array}$ & & $\begin{array}{l}\mathrm{AT}(\mathrm{ml} / \mathrm{kg} / \mathrm{min}) \\
\mathrm{V}_{\mathrm{E}} / \mathrm{V}_{\mathrm{CO} 2}\end{array}$ & $\begin{array}{l}\text { Cardiopulmonary } \\
\text { complication }\end{array}$ & $\begin{array}{l}\text { Acute myocardial infarction (detection of a } \\
\text { rise in serum troponin) and } \\
\text { electrocardiographic changes indicative } \\
\text { of new ischemia; congestive cardiac } \\
\text { failure; and serious dysrhythmia } \\
\text { resulting in compromised tissue } \\
\text { perfusion and primary cardiac arrest. } \\
\text { Pneumonia, pleural effusion, and } \\
\text { respiratory failure requiring ventilatory } \\
\text { support }\end{array}$ \\
\hline & & & & & Mortality & $\begin{array}{l}\text { In-hospital death and death within } 30 \text { days } \\
\text { from surgery }\end{array}$ \\
\hline West 2014 & Rectal & $\begin{array}{l}\text { Mean age (SD): } 66 \\
\quad(10) \\
\text { Sample size: } 95 \\
\text { Female }(\%): 23 \\
\quad(24 \%)\end{array}$ & Cycloergometer & $\begin{array}{l}\text { Peak VO2 }(\mathrm{ml} / \\
\mathrm{kg} / \mathrm{min}) \\
\mathrm{V}_{\mathrm{E}} / \mathrm{V}_{\mathrm{CO} 2}\end{array}$ & $\begin{array}{l}\text { Postoperative } \\
\text { complication }\end{array}$ & $\begin{array}{l}\text { Postoperative Morbidity Survey (POMS) } \\
\text { on Day 5, the Dindo-Demartines- } \\
\text { Clavien classification (highest grade for } \\
\text { the most serious sustained in-hospital } \\
\text { morbidity) and in-hospital mortality } \\
\text { were recorded }\end{array}$ \\
\hline
\end{tabular}

preoperative $\mathrm{V}_{\mathrm{E}} / \mathrm{V}_{\mathrm{CO} 2}$ values were observed for patients with or without postoperative complication (MD: 0.80; 95\% CI: -0.95 to 2.54 ) and minor or major postoperative complication (MD: 0.93 ; 95\% CI: -1.53 to 3.38 ) (Fig. 4). Other studies were not pooled in the meta-analysis due to heterogeneity and reported mixed results (Tables 3 and 4).

\section{Association between Preoperative CPET Values and Length of Hospital Stay}

The association between preoperative Peak $\mathrm{VO}_{2}(7$ studies), AT (8 studies), and $\mathrm{V}_{\mathrm{E}} / \mathrm{V}_{\mathrm{CVO} 2}$ (3 studies) and length of hospital stay is presented in Table 5. Results of individual studies provided mixed results. Some studies reported a positive association between CPET variables and length of hospital stay (i.e., patients presenting higher CPET values stayed shorter in hospital), and others reported no statistical differences. However, none of the 
TABLE 2 Risk of bias assessment using the Quality in Prognosis Studies (QUIPS) tool

\begin{tabular}{|c|c|c|c|c|}
\hline Author, year & Study participation & Study attrition & Outcome measurement & Statistical analysis and reporting \\
\hline Lamb 2016 & Low & Low & Low & Moderate \\
\hline Prentis 2013 & Moderate & Low & Low & Low \\
\hline Tolchard 2015 & Moderate & Low & Low & Low \\
\hline Bowles 2008 & Moderate & Low & Low & Low \\
\hline Chan 2016 & Low & Low & Low & Moderate \\
\hline Mann 2020 & Low & Low & Low & Low \\
\hline McSorley 2018/Stephen 2018 & Moderate & Low & Moderate & Moderate \\
\hline Nikolopoulos 2015 & Moderate & Low & Low & Low \\
\hline Forshaw 2008 & Low & Low & Low & Low \\
\hline Lam 2019 & Low & Low & Low & Low \\
\hline Nagamatsu 2001/Nagamatsu 1994 & Low & Low & Low & Low \\
\hline Patel 2019 & Low & Low & Low & Moderate \\
\hline Sinclair 2017 & Low & Low & Low & Low \\
\hline Dunne 2014 & Moderate & High & Low & Low \\
\hline Kasivisvanathan 2015 & Low & Moderate & Low & Low \\
\hline Ulyett 2017 & Low & Moderate & Low & Low \\
\hline Bayram 2007 & Low & Low & Low & Low \\
\hline Bechard \& Wetstein 1987 & Moderate & Low & Low & Low \\
\hline Bobbio 2009 & Moderate & Low & Low & Low \\
\hline Bolliger 1995/ Bolliger 1996 & Low & Low & Low & Low \\
\hline Brat 2016 & Low & Low & Low & Low \\
\hline Brunelli 2009 & Moderate & Low & Low & Low \\
\hline Brunelli 2012 & Low & Low & Low & Low \\
\hline Brutsche 2000 & Low & Low & Low & Low \\
\hline Dales 1993 & Moderate & Low & Low & Low \\
\hline Epstein 1993 & Low & Low & Low & Low \\
\hline Fang 2014 & Moderate & Low & Moderate & Low \\
\hline Han 2007 & Low & Low & Low & Low \\
\hline Kasikcioglu 2009 & Moderate & Low & Low & Low \\
\hline Licker 2011 & Moderate & Low & Low & Low \\
\hline Loewen 2007 & Low & Low & Low & Low \\
\hline Mao 2010 & Moderate & Low & Low & Low \\
\hline Markos 1989 & Low & Low & Low & Low \\
\hline Miyazki 2018 & Moderate & Moderate & Low & Low \\
\hline Morice 1992 & Moderate & Low & Low & Low \\
\hline Nagamatsu 2004/Nagamatsu 2005 & Moderate & Low & Low & Low \\
\hline Pate 1996 & Low & Low & Low & Low \\
\hline Rodrigues 2016 & Moderate & Low & Moderate & Low \\
\hline Torchio 2010/Torchio 2017 & Low & Low & Low & Low \\
\hline Villani \& Busia 2004 & Low & Low & Low & Low \\
\hline Win 2005 & Low & Low & Low & Low \\
\hline Yakal 2018 & Moderate & Low & Moderate & Low \\
\hline Begum 2016 & Low & Low & Low & Low \\
\hline Huang 2016 & Low & Low & Low & Low \\
\hline Moyes 2013 Drummond 2018 & Moderate & Low & Low & Low \\
\hline Snowden 2013 & Moderate & Low & Low & Low \\
\hline Whibley 2018 & Moderate & Low & Moderate & Moderate \\
\hline Wilson 2010 & Moderate & Low & Low & Moderate \\
\hline
\end{tabular}


TABLE 2 continued

\begin{tabular}{lllll}
\hline Author, year & Study participation & Study attrition & Outcome measurement & Statistical analysis and reporting \\
\hline Ausania 2012 & Low & Low & Low & Moderate \\
Chandrabalan 2013 & High & Low & Low & Low \\
Junejo 2014 & Low & Moderate & Low & Low \\
West 2014 & Low & Moderate & Low & Low \\
\hline
\end{tabular}

studies reported a significant negative association (i.e., patients presenting lower CPET values stayed for shorter periods in hospital) (Table 5).

\section{Association between Preoperative CPET Values and Postoperative Quality of Life}

Currently, no study has investigated the association between preoperative CPET values and postoperative quality of life outcomes in patients undergoing cancer surgery.

\section{DISCUSSION}

\section{Statement of Principal Findings}

This systematic review identified many studies investigating the potential association between preoperative CPET values and postoperative complications and length of hospital stay. Our meta-analysis demonstrated that higher preoperative Peak $\mathrm{VO}_{2}, \mathrm{AT}$, and lower $\mathrm{V}_{\mathrm{E}} / \mathrm{V}_{\mathrm{CO} 2}$ values were predominately significantly associated with absence of postoperative complications. Several individual studies were not included in the meta-analysis due to heterogeneity in the CPET values and outcomes or did not report appropriate values to be pooled. While the results of individual studies provided mixed results, it is important to note that none reported a negative association (i.e., superior preoperative CPET values associated with worst postoperative outcome). Similarly, the association between preoperative CPET values and length of hospital stay reported in individual studies provided mixed results; none reported a negative association. Interestingly, this review was not able to identify any study investigating the association between preoperative CPET values and postoperative quality of life outcomes.

\section{Strengths and Weaknesses of the Study}

The strengths of this systematic review and meta-analyses were the methodology employed, following recommendation from the Cochrane Prognosis Review Group, and were reported according to the MOOSE framework. In addition, we conducted a sensitive search on major medical databases, that was supported by a senior librarian. Our search was only limited by human subjects and included all the literature irrespective of language and publication year. Furthermore, we assessed risk of bias using a well stablished tool (QUIPS).

The limitation of our systematic review included the heterogeneity between the included studies. For many included studies, meta-analysis was not possible as the CPET variables and outcome measures were not standardised and prevented pooling of the data. Also, due to the population of interest (patients undergoing cancer surgery), peak $\mathrm{VO}_{2}$ and $\mathrm{VO}_{2}$ max were used in this review interchangeably. ${ }^{6}$ Because these patients are older and debilitated by their conditions, it is difficult to demonstrate that the plateau criterion for $\mathrm{VO}_{2}$ max has been met in response to exercise. Furthermore, none of the included studies investigated the association between preoperative CPET and postoperative quality of life, underpinning the lack of evidence for this important patient reported outcome. Lastly, while we included a large number of full-text manuscripts published in scientific journals, we excluded studies that were published as abstracts of conference proceedings.

\section{Comparison with Other Studies}

The association between preoperative CPET variables and postoperative complications and/or length of hospital stay has been investigated in previous systematic reviews, reporting mixed results. While there are few systematic reviews indicating a positive association between superior preoperative CPET values and absence of postoperative complications, others reported no significant association. $5,7,8,10,11$ This is somewhat in line with the results of the current review. Our meta-analysis showed that superior preoperative CPET values are significantly associated with the absence of most postoperative complications. However, results from studies that were not included in our metaanalysis are somewhat less favorable.

Despite this, there are some differences between the current and previous systematic reviews that are important to note. Previous systematic reviews included a smaller 
Author, year $\quad$ Type of cancer Mean difference (95\%CI)

Mean difference $(95 \% \mathrm{CI})$

Weight (\%)

No complication vs Complication

$\begin{array}{llc}\text { Bolliger, 1995 } & \text { Lung } & 3.40(0.44 \text { to } 6.36) \\ \text { Brunelli, 2009 } & \text { Lung } & 1.15(-0.10 \text { to } 2.40) \\ \text { Brutsche, 2000 } & \text { Lung } & 4.20(2.01 \text { to } 6.39) \\ \text { Epstein, 1993 } & \text { Lung } & 0.30(-2.80 \text { to } 3.40) \\ \text { Fang, 2014 } & \text { Lung } & 3.50(1.51 \text { to } 5.49) \\ \text { Licker, 2011 } & \text { Lung } & 3.90(2.25 \text { to } 5.55) \\ \text { Kasikeioglu, 2009 } & \text { Lung } & 3.70(1.58 \text { to } .82) \\ \text { Lam, 2019 } & \text { Esophageal } & -0.40(-1.63 \text { to } 0.83) \\ \text { Prentis, 2013 } & \text { Bladder } & 0.10(-2.04 \text { to } 2.24) \\ \text { Pate, 1996 } & \text { Lung } & -0.43(-3.12 \text { to } 2.28) \\ \text { West, 2014 } & \text { Rectal } & 6.00(3.98 \text { to } 8.02) \\ \text { Win, 2005 } & \text { Lung } & 1.70(-0.36 \text { to } 3.78) \\ \text { Yakal, 2018 } & \text { Lung } & 1.14(0.34 \text { to } 2.62) \\ \text { Bechand and Welstein, 1987 } & \text { Lung } & 7.06(3.53 \text { to } 10.59) \\ \text { Villani and Busia, 2004 } & \text { Lung } & 1.60(0.29 \text { to } 2.91) \\ \text { Markos, 1989 } & \text { Lung } & 0.70(-3.14 \text { to } 4.54) \\ \text { Pooled effect }\left(I^{2}=9 \%\right) & & 2.28(1.26 \text { to } 3.29) \\ \text { lmonary complication vs Pulmonary complication } & \\ \text { Bayram, 2007 } & \text { Lung } & 1.90(0.41 \text { to } 3.39) \\ \text { Bobbio, 2009 } & \text { Lung } & 3.30(1.03 \text { to } 5.57) \\ \text { Brat, 2016 } & \text { Lung } & 7.20(-2.86 \text { to } 17.26) \\ \text { Brunelli, 2009 } & \text { Lung } & 2.80(0.24 \text { to } 5.36) \\ \text { Licker, 2011 } & \text { Lung } & 1.20(-1.23 \text { to } 3.63) \\ \text { Brunelli, 2012 } & \text { Lung } & 0.50(-1.06 \text { to } 2.08) \\ \text { Han, 2007 } & \text { Lung } & -0.10(-1.39 \text { to } 1.19) \\ \text { Morice, 1992 } & \text { Lung } & 2.00(-0.96 \text { to } 4.98) \\ \left.\text { Pooled effect (I }{ }^{2}=0 \%\right) & & 1.47(0.49 \text { to } 2.45)\end{array}$

No cardiopulmonary complication vs Cardiopulmonary complication

$\begin{array}{llr}\text { Forshaw, 2008 } & \text { Esophageal } & -0.90(-2.15 \text { to } 0.35) \\ \text { Lam, 2019 } & \text { Esophageal } & 2.20(-0.01 \text { to } 4.41) \\ \text { Moyes, 2013 } & \text { Mixed } & 2.00(-0.28 \text { to } 4.28) \\ \begin{array}{l}\text { Redrigues, } 2016 \\ \text { Pooled effect }\left(\mathrm{I}^{2}=0 \%\right)\end{array} & \text { Lung } & 0.85(-0.71 \text { to } 2.41) \\ \text { complication vs Major complication } & 0.84(-0.63 \text { to } 2.35) \\ \text { Tolchand, 2015 } & \text { Bladder } & 1.85(0.70 \text { to } 3.00) \\ \text { Terchio, 2017 } & \text { Lung } & 1.80(0.73 \text { to } 2.87) \\ \text { Chan, 2016 } & \text { Colorectal } & 3.53(0.84 \text { to } 6.22) \\ \text { Patel, 2019 } & \text { Esophageal } & 4.16(1.61 \text { to } 6.71) \\ \text { Ulyett, 2017 } & \text { Liver } & -0.40(-2.75 \text { to } 1.95) \\ \text { Pooled effect }\left(\mathrm{I}^{2}=27 \%\right) & & 2.01(0.90 \text { to } 3.13)\end{array}$

No cardiovascular complication vs Cardiovascular complication

$\begin{array}{lll}\text { Licker, } 2011 & \text { Lung } & 3.50(1.29 \text { to } 5.71) \\ \text { Loewen, } 2007 & \text { Lung } & 1.47(0.55 \text { to } 2.39) \\ \text { Pooled effect }\left(\mathrm{I}^{2}=0 \%\right) & & 2.23(0.30 \text { to } 4.15)\end{array}$

No mortality vs Mortality (In-hospital)

$\begin{array}{lll}\text { Brunelli, } 2009 & \text { Lung } & 3.70(0.63 \text { to } 6.77) \\ \text { Snowden, } 2013 & \text { Mixed } & 2.40(0.44 \text { to } 4.36) \\ \text { Pooled effect }\left(I^{2}=0 \%\right) & & 2.78(1.12 \text { to } 4.43)\end{array}$

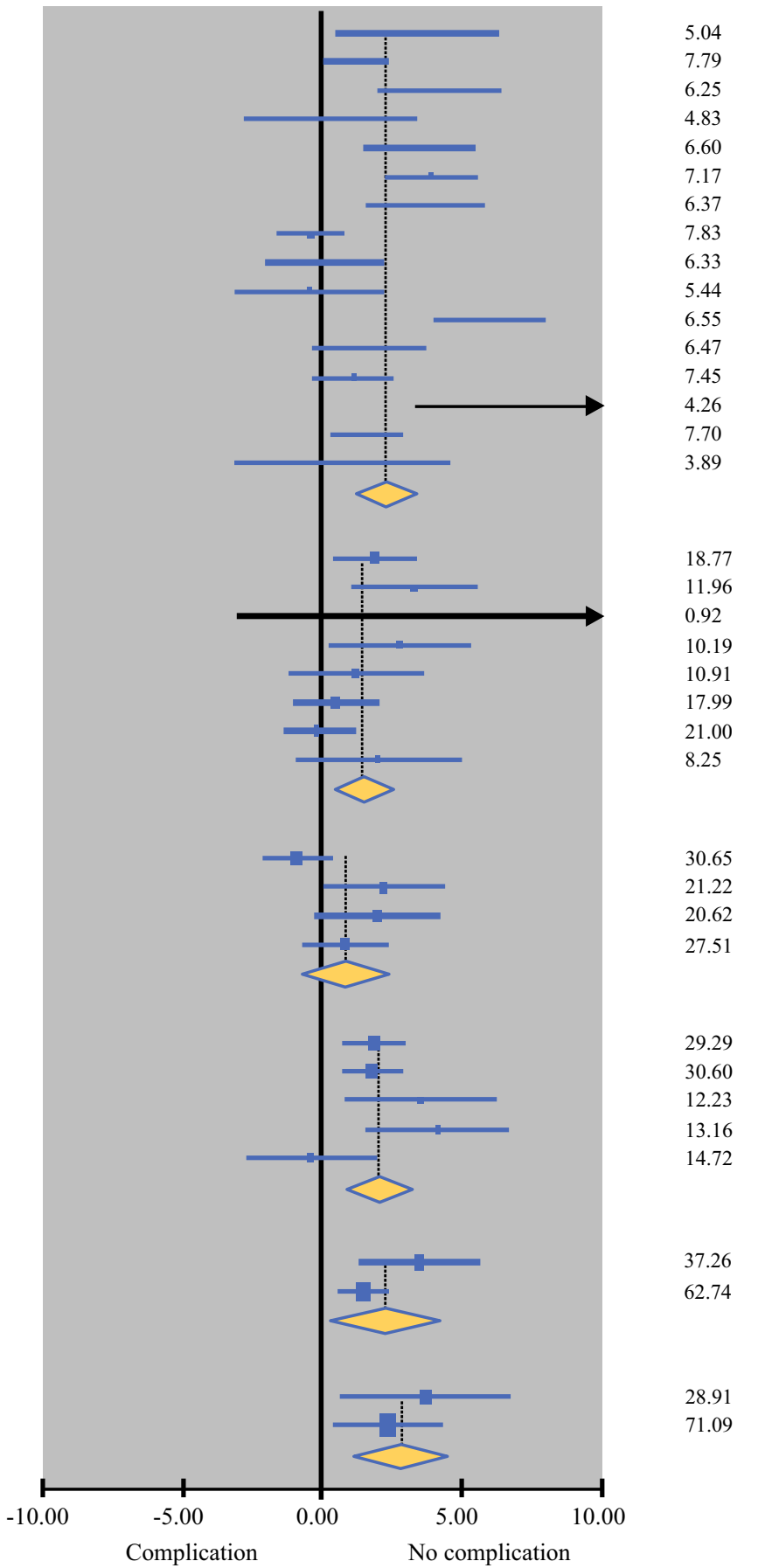

FIG. 2 Forest plot of the association between preoperative peak oxygen uptake (peak $\mathrm{VO}_{2}$ ) in $\mathrm{ml} / \mathrm{kg} / \mathrm{min}$ and postoperative complication. Mean difference $>0$ indicate higher preoperative peak $\mathrm{VO}_{2}$ in patients with no postoperative complications. $\mathrm{CI}=\mathrm{Confidence}$ level

number of studies (ranging from 7-37), investigated postoperative complications as the main outcome measure, and included either a specific cancer population undergoing surgery (e.g., lung, esophageal), or mixed populations undergoing surgery for cancer and/or noncancer related conditions. ${ }^{5,71}$ Meta-analysis was attempted in only half of the previous published systematic reviews. Therefore, the mixed results encountered between the current review and previous reviews may be because the inclusion and exclusion criterion were different. The heterogeneity of the included cohorts, including the lack of consistency in reporting or standardisation of outcomes were highlighted 
TABLE 3 Association between preoperative cardiopulmonary exercise test variables and postoperative complication

\begin{tabular}{|c|c|c|c|c|c|c|c|}
\hline \multirow[t]{2}{*}{ Author, year } & \multirow{2}{*}{$\begin{array}{l}\text { Cancer } \\
\text { type }(\mathrm{N})\end{array}$} & \multicolumn{2}{|c|}{ Preoperative CPET threshold } & \multicolumn{2}{|c|}{ Postoperative complication } & \multirow{2}{*}{$\begin{array}{l}\text { Estimates, odds ratio ( } 95 \% \\
\text { confidence intervals) }\end{array}$} & \multirow[t]{2}{*}{ Summary } \\
\hline & & Favorable & Unfavorable & Favorable outcome & $\begin{array}{l}\text { Unfavorable } \\
\text { outcome }\end{array}$ & & \\
\hline Licker 2011 & & $\begin{array}{l}\text { Peak } \mathrm{VO}_{2}>10 \\
\quad(\mathrm{~mL} / \mathrm{Kg} / \mathrm{min})\end{array}$ & $\begin{array}{l}\text { Peak } \mathrm{VO}_{2} \leq 10 \\
(\mathrm{~mL} / \mathrm{Kg} / \mathrm{min})\end{array}$ & No complication & Complication & $0.19(0.09-0.42)$ & \\
\hline West 2014 & Rectal (95) & $\begin{array}{l}\text { Peak } \mathrm{VO}_{2} \geq 10.6 \\
\quad(\mathrm{~mL} / \mathrm{Kg} / \mathrm{min})\end{array}$ & $\begin{array}{c}\text { Peak } \mathrm{VO}_{2}<10.6 \\
(\mathrm{~mL} / \mathrm{Kg} / \mathrm{min})\end{array}$ & No complication & Complication & $0.02(0.01-0.10)$ & \\
\hline Bayram 2007 & Lung (55) & $\begin{array}{l}\text { Peak } \mathrm{VO}_{2}>15 \\
(\mathrm{~mL} / \mathrm{Kg} / \mathrm{min})\end{array}$ & $\begin{array}{l}\text { Peak } \mathrm{VO}_{2}<15 \\
(\mathrm{~mL} / \mathrm{Kg} / \mathrm{min})\end{array}$ & No complication & Complication & $0.47(0.15-1.46)$ & \\
\hline Epstein 1993 & Lung (42) & $\begin{array}{l}\text { Peak } \mathrm{VO}_{2}>15 \\
\quad(\mathrm{~mL} / \mathrm{Kg} / \mathrm{min})\end{array}$ & $\begin{array}{l}\text { Peak } \mathrm{VO}_{2}<15 \\
(\mathrm{~mL} / \mathrm{Kg} / \mathrm{min})\end{array}$ & No complication & Complication & $0.42(0.11-1.55)$ & \\
\hline Licker 2011 & Lung (215) & $\begin{array}{l}\text { Peak } \mathrm{VO}_{2} \geq 17 \\
\quad(\mathrm{~mL} / \mathrm{Kg} / \mathrm{min})\end{array}$ & $\begin{array}{l}\text { Peak } \mathrm{VO}_{2}<17 \\
(\mathrm{~mL} / \mathrm{Kg} / \mathrm{min})\end{array}$ & No complication & Complication & $0.35(0.17-0.73)$ & \\
\hline McSorley 2018 & $\begin{array}{l}\text { Colorectal } \\
\quad(38)\end{array}$ & $\begin{array}{l}\text { Peak } \mathrm{VO}_{2}>19 \\
\quad(\mathrm{~mL} / \mathrm{Kg} / \mathrm{min})\end{array}$ & $\begin{array}{l}\text { Peak } \mathrm{VO}_{2}<19 \\
(\mathrm{~mL} / \mathrm{Kg} / \mathrm{min})\end{array}$ & No complication & Complication & $0.94(0.24-3.71)$ & \\
\hline Dales 1993 & Lung (46) & $\begin{array}{l}\text { Peak } \mathrm{VO}_{2} \geq 1250 \\
\quad(\mathrm{ml})\end{array}$ & $\begin{array}{l}\text { Peak } \mathrm{VO}_{2}<1250 \\
\quad(\mathrm{ml})\end{array}$ & No complication & Complication & $0.27(0.08-0.93)$ & \\
\hline Epstein 1993 & Lung (42) & Peak $\mathrm{VO}_{2}>1(\mathrm{~L})$ & Peak $\mathrm{VO}_{2} \leq 1(\mathrm{~L})$ & No complication & Complication & $0.33(0.09-1.29)$ & \\
\hline Epstein 1993 & Lung (42) & $\begin{array}{l}\text { Peak } \mathrm{VO}_{2}>500 \\
\left(\mathrm{ml} / \mathrm{m}^{2}\right)\end{array}$ & $\begin{array}{l}\text { Peak } \mathrm{VO}_{2} \leq 500 \\
\left(\mathrm{ml} / \mathrm{m}^{2}\right)\end{array}$ & No complication & Complication & $0.17(0.04-0.74)$ & \\
\hline Chan 2016 & $\begin{array}{l}\text { Colorectal } \\
\quad(48)\end{array}$ & Peak $\mathrm{VO}_{2}(\mathrm{~mL} / \mathrm{Kg}$ & & No complication & Complication & NR & \\
\hline Dunne 2014 & Liver (197) & Peak $\mathrm{VO}_{2}(\mathrm{~mL} / \mathrm{Kg}$ & & No complication & Complication & $1.02(0.96-1.09)$ & \\
\hline Junejo 2014 & $\begin{array}{l}\text { Pancreas } \\
\text { (64) }\end{array}$ & Peak $\mathrm{VO}_{2}(\mathrm{~mL} / \mathrm{Kg}$ & & No complication & Complication & $1.00(0.86-1.18)$ & \\
\hline Sinclair 2017 & $\begin{array}{l}\text { Esophagus } \\
(240)\end{array}$ & Peak $\mathrm{VO}_{2}(\mathrm{ml})$ & & No complication & Complication & $1.00(1.00-1.00)$ & \\
\hline $\begin{array}{l}\text { Kasivisvanathan } \\
\quad 2015\end{array}$ & Liver (104) & Peak $\mathrm{VO}_{2}(\mathrm{~mL} / \mathrm{Kg}$ & in) & No complication & Complication & $1.03(1.01-1.06)$ & \\
\hline $\begin{array}{l}\text { Nagamatsu } \\
\text { 2001/Nagamatsu } \\
1994\end{array}$ & $\begin{array}{l}\text { Esophagus } \\
\text { (91) }\end{array}$ & $\begin{array}{l}\text { Peak } \mathrm{VO}_{2} \geq 1000 \\
\quad\left(\mathrm{ml} / \mathrm{min} / \mathrm{m}^{2}\right)\end{array}$ & $\begin{array}{c}\text { Peak } \mathrm{VO}_{2}<1000 \\
\left(\mathrm{ml} / \mathrm{min} / \mathrm{m}^{2}\right)\end{array}$ & $\begin{array}{l}\text { No cardiopulmonary } \\
\text { complication }\end{array}$ & $\begin{array}{l}\text { Cardiopulmonary } \\
\text { complication }\end{array}$ & $0.22(0.05-1.03)$ & \\
\hline Miyazaki 2018 & Lung (209) & $\begin{array}{l}\text { Peak } \mathrm{VO}_{2} \geq 12 \\
\quad(\mathrm{~mL} / \mathrm{Kg} / \mathrm{min})\end{array}$ & $\begin{array}{l}\text { Peak } \mathrm{VO}_{2}<12 \\
\quad(\mathrm{~mL} / \mathrm{Kg} / \mathrm{min})\end{array}$ & $\begin{array}{l}\text { No cardiopulmonary } \\
\text { complication }\end{array}$ & $\begin{array}{l}\text { Cardiopulmonary } \\
\text { complication }\end{array}$ & $0.62(0.24-1.59)$ & \\
\hline Bayram 2007 & Lung (55) & $\begin{array}{l}\text { Peak } \mathrm{VO}_{2}>15 \\
\quad(\mathrm{~mL} / \mathrm{Kg} / \mathrm{min})\end{array}$ & $\begin{array}{l}\text { Peak } \mathrm{VO}_{2}<15 \\
\quad(\mathrm{~mL} / \mathrm{Kg} / \mathrm{min})\end{array}$ & $\begin{array}{l}\text { No cardiopulmonary } \\
\text { complication }\end{array}$ & $\begin{array}{c}\text { Cardiopulmonary } \\
\text { complication }\end{array}$ & $0.07(0.01-0.35)$ & \\
\hline
\end{tabular}


TABLE 3 continued

\begin{tabular}{|c|c|c|c|c|c|c|c|}
\hline \multirow[t]{2}{*}{ Author, year } & \multirow{2}{*}{$\begin{array}{l}\text { Cancer } \\
\text { type }(\mathrm{N})\end{array}$} & \multicolumn{2}{|c|}{ Preoperative CPET threshold } & \multicolumn{2}{|c|}{ Postoperative complication } & \multirow{2}{*}{$\begin{array}{l}\text { Estimates, odds ratio ( } 95 \% \\
\text { confidence intervals) }\end{array}$} & \multirow[t]{2}{*}{ Summary } \\
\hline & & Favorable & Unfavorable & Favorable outcome & $\begin{array}{l}\text { Unfavorable } \\
\text { outcome }\end{array}$ & & \\
\hline Mao 2010 & Lung (198) & $\begin{array}{l}\text { Peak } \mathrm{VO}_{2}>15(\mathrm{~mL} / \\
\mathrm{Kg} / \mathrm{min})\end{array}$ & $\begin{array}{l}\text { Peak } \mathrm{VO}_{2}<15(\mathrm{~mL} / \\
\mathrm{Kg} / \mathrm{min})\end{array}$ & $\begin{array}{l}\text { No cardiopulmonary } \\
\text { complication }\end{array}$ & $\begin{array}{l}\text { Cardiopulmonary } \\
\text { complication }\end{array}$ & $0.23(0.08-0.62)$ & \\
\hline $\begin{array}{c}\text { Miyazaki } \\
2018\end{array}$ & Lung (209) & $\begin{array}{l}\text { Peak } \mathrm{VO}_{2}>15(\mathrm{~mL} / \\
\mathrm{Kg} / \mathrm{min})\end{array}$ & $\begin{array}{l}\text { Peak } \mathrm{VO}_{2}<15(\mathrm{~mL} / \\
\mathrm{Kg} / \mathrm{min})\end{array}$ & $\begin{array}{l}\text { No cardiopulmonary } \\
\text { complication }\end{array}$ & $\begin{array}{l}\text { Cardiopulmonary } \\
\text { complication }\end{array}$ & $0.88(0.49-1.60)$ & \\
\hline Begum 2016 & $\begin{array}{l}\text { Lung } \\
\qquad(1684)\end{array}$ & $\begin{array}{l}\text { Peak } \mathrm{VO}_{2}>15(\mathrm{~mL} / \\
\mathrm{Kg} / \mathrm{min})\end{array}$ & $\begin{array}{l}\text { Peak } \mathrm{VO}_{2}<15(\mathrm{~mL} / \\
\mathrm{Kg} / \mathrm{min})\end{array}$ & $\begin{array}{l}\text { No cardiopulmonary } \\
\text { complication }\end{array}$ & $\begin{array}{l}\text { Cardiopulmonary } \\
\text { complication }\end{array}$ & $0.82(0.65-1.03)$ & \\
\hline Mao 2010 & Lung (198) & $\begin{array}{l}\text { Peak } \mathrm{VO}_{2} \geq 20(\mathrm{~mL} / \\
\mathrm{Kg} / \mathrm{min})\end{array}$ & $\begin{array}{l}\text { Peak } \mathrm{VO}_{2}<20(\mathrm{~mL} / \\
\mathrm{Kg} / \mathrm{min})\end{array}$ & $\begin{array}{l}\text { No cardiopulmonary } \\
\text { complication }\end{array}$ & $\begin{array}{l}\text { Cardiopulmonary } \\
\text { complication }\end{array}$ & $0.56(0.29-1.09)$ & \\
\hline Junejo 2014 & $\begin{array}{l}\text { Pancreas } \\
\text { (64) }\end{array}$ & Peak $\mathrm{VO}_{2}(\mathrm{~mL} / \mathrm{Kg} / \mathrm{min}$ & & $\begin{array}{l}\text { No cardiopulmonary } \\
\text { complication }\end{array}$ & $\begin{array}{l}\text { Cardiopulmonary } \\
\text { complication }\end{array}$ & $1.00(0.86-1.17)$ & \\
\hline Sinclair 2017 & $\begin{array}{l}\text { Esophagus } \\
\quad(240)\end{array}$ & Peak $\mathrm{VO}_{2}(\mathrm{ml})$ & & $\begin{array}{l}\text { No cardiopulmonary } \\
\text { complication }\end{array}$ & $\begin{array}{l}\text { Cardiopulmonary } \\
\text { complication }\end{array}$ & $0.99(0.99-1.00)$ & \\
\hline West 2014 & Rectal (95) & $\begin{array}{l}\text { Peak } \mathrm{VO}_{2} \geq 10.6 \\
\quad(\mathrm{~mL} / \mathrm{Kg} / \mathrm{min})\end{array}$ & $\begin{array}{c}\text { Peak } \mathrm{VO}_{2}<10.6 \\
(\mathrm{~mL} / \mathrm{Kg} / \mathrm{min})\end{array}$ & $\begin{array}{l}\text { No pulmonary } \\
\text { complication }\end{array}$ & $\begin{array}{l}\text { Pulmonary } \\
\text { complication }\end{array}$ & $0.09(0.02-0.46)$ & \\
\hline Bayram 2007 & Lung (55) & $\begin{array}{l}\text { Peak } \mathrm{VO}_{2}>15(\mathrm{~mL} / \\
\mathrm{Kg} / \mathrm{min})\end{array}$ & $\begin{array}{l}\text { Peak } \mathrm{VO}_{2}<15(\mathrm{~mL} / \\
\mathrm{Kg} / \mathrm{min})\end{array}$ & $\begin{array}{l}\text { No pulmonary } \\
\text { complication }\end{array}$ & $\begin{array}{l}\text { Pulmonary } \\
\text { complication }\end{array}$ & $0.03(0.00-0.53)$ & \\
\hline Licker 2011 & Lung (215) & $\begin{array}{l}\text { Peak } \mathrm{VO}_{2}>10(\mathrm{~mL} / \\
\mathrm{Kg} / \mathrm{min})\end{array}$ & $\begin{array}{l}\text { Peak } \mathrm{VO}_{2} \leq 10(\mathrm{~mL} / \\
\mathrm{Kg} / \mathrm{min})\end{array}$ & $\begin{array}{l}\text { No cardiovascular } \\
\text { complication }\end{array}$ & $\begin{array}{l}\text { Cardiovascular } \\
\text { complication }\end{array}$ & $0.25(0.10-0.63)$ & \\
\hline West 2014 & Rectal (95) & $\begin{array}{c}\text { Peak } \mathrm{VO}_{2} \geq 10.6 \\
(\mathrm{~mL} / \mathrm{Kg} / \mathrm{min})\end{array}$ & $\begin{array}{c}\text { Peak } \mathrm{VO}_{2}<10.6 \\
(\mathrm{~mL} / \mathrm{Kg} / \mathrm{min})\end{array}$ & $\begin{array}{l}\text { No cardiovascular } \\
\text { complication }\end{array}$ & $\begin{array}{l}\text { Cardiovascular } \\
\text { complication }\end{array}$ & $0.17(0.03-0.87)$ & \\
\hline Licker 2011 & Lung (215) & $\begin{array}{l}\text { Peak } \mathrm{VO}_{2} \geq 17(\mathrm{~mL} / \\
\mathrm{Kg} / \mathrm{min})\end{array}$ & $\begin{array}{l}\text { Peak } \mathrm{VO}_{2}<17(\mathrm{~mL} / \\
\mathrm{Kg} / \mathrm{min})\end{array}$ & $\begin{array}{l}\text { No cardiovascular } \\
\text { complication }\end{array}$ & $\begin{array}{l}\text { Cardiovascular } \\
\text { complication }\end{array}$ & $0.42(0.16-1.09)$ & \\
\hline Dales 1993 & Lung (46) & $\begin{array}{l}\text { Peak } \mathrm{VO}_{2} \geq 1250 \\
\quad(\mathrm{~mL} / \mathrm{Kg} / \mathrm{min})\end{array}$ & $\begin{array}{l}\text { Peak } \mathrm{VO}_{2}<1250 \\
\quad(\mathrm{~mL} / \mathrm{Kg} / \mathrm{min})\end{array}$ & $\begin{array}{l}\text { No respiratory } \\
\text { complication }\end{array}$ & $\begin{array}{l}\text { Respiratory } \\
\text { complication }\end{array}$ & $0.24(0.06-0.88)$ & \\
\hline Licker 2011 & Lung (215) & $\begin{array}{c}\text { Peak } \mathrm{VO}_{2}>10(\mathrm{~mL} / \\
\mathrm{Kg} / \mathrm{min})\end{array}$ & $\begin{array}{l}\text { Peak } \mathrm{VO}_{2} \leq 10(\mathrm{~mL} / \\
\mathrm{Kg} / \mathrm{min})\end{array}$ & $\begin{array}{l}\text { No respiratory } \\
\text { complication }\end{array}$ & $\begin{array}{l}\text { Respiratory } \\
\text { complication }\end{array}$ & $0.28(0.12-0.68)$ & \\
\hline Whibley 2018 & Mixed (81) & $\begin{array}{l}\text { Peak } \mathrm{VO}_{2} \geq 14(\mathrm{~mL} / \\
\mathrm{Kg} / \mathrm{min})\end{array}$ & $\begin{array}{l}\text { Peak } \mathrm{VO}_{2}<14(\mathrm{~mL} / \\
\mathrm{Kg} / \mathrm{min})\end{array}$ & $\begin{array}{l}\text { No respiratory } \\
\text { complication }\end{array}$ & $\begin{array}{l}\text { Respiratory } \\
\text { complication }\end{array}$ & NR & \\
\hline Bayram 2007 & Lung (55) & $\begin{array}{l}\text { Peak } \mathrm{VO}_{2}>15(\mathrm{~mL} / \\
\mathrm{Kg} / \mathrm{min})\end{array}$ & $\begin{array}{l}\text { Peak } \mathrm{VO}_{2}<15(\mathrm{~mL} / \\
\mathrm{Kg} / \mathrm{min})\end{array}$ & $\begin{array}{l}\text { No respiratory } \\
\text { complication }\end{array}$ & $\begin{array}{l}\text { Respiratory } \\
\text { complication }\end{array}$ & $0.51(0.02-15.84)$ & \\
\hline Licker 2011 & Lung (215) & $\begin{array}{l}\text { Peak } \mathrm{VO}_{2} \geq 17(\mathrm{~mL} / \\
\mathrm{Kg} / \mathrm{min})\end{array}$ & $\begin{array}{l}\text { Peak } \mathrm{VO}_{2}<17(\mathrm{~mL} / \\
\mathrm{Kg} / \mathrm{min})\end{array}$ & $\begin{array}{l}\text { No respiratory } \\
\text { complication }\end{array}$ & $\begin{array}{l}\text { Respiratory } \\
\text { complication }\end{array}$ & $0.40(0.16-0.95)$ & \\
\hline Bayram 2007 & Lung (55) & $\begin{array}{l}\text { Peak } \mathrm{VO}_{2}>15(\mathrm{~mL} / \\
\mathrm{Kg} / \mathrm{min})\end{array}$ & $\begin{array}{l}\text { Peak } \mathrm{VO}_{2}<15(\mathrm{~mL} / \\
\mathrm{Kg} / \mathrm{min})\end{array}$ & No mortality (30 days) & Mortality (30 days) & $0.20(0.01-5.7)$ & \\
\hline
\end{tabular}


TABLE 3 continued

\begin{tabular}{|c|c|c|c|c|c|c|c|}
\hline \multirow[t]{2}{*}{ Author, year } & \multirow{2}{*}{$\begin{array}{l}\text { Cancer } \\
\text { type }(\mathrm{N})\end{array}$} & \multicolumn{2}{|c|}{ Preoperative CPET threshold } & \multicolumn{2}{|c|}{ Postoperative complication } & \multirow{2}{*}{$\begin{array}{l}\text { Estimates, odds ratio (95\% } \\
\text { confidence intervals) }\end{array}$} & \multirow[t]{2}{*}{ Summary } \\
\hline & & Favorable & Unfavorable & Favorable outcome & $\begin{array}{l}\text { Unfavorable } \\
\text { outcome }\end{array}$ & & \\
\hline Begum 2016 & $\begin{array}{l}\text { Lung } \\
\qquad(1684)\end{array}$ & $\begin{array}{l}\text { Peak } \mathrm{VO}_{2}>15(\mathrm{~mL} / \\
\mathrm{Kg} / \mathrm{min})\end{array}$ & $\begin{array}{l}\text { Peak } \mathrm{VO}_{2}<15(\mathrm{~mL} / \\
\mathrm{Kg} / \mathrm{min})\end{array}$ & $\begin{array}{l}\text { No mortality ( } 30 \\
\text { days) }\end{array}$ & Mortality (30 days) & $0.60(0.35-0.93)$ & \\
\hline Junejo 2014 & $\begin{array}{l}\text { Pancreatic } \\
\text { (64) }\end{array}$ & \multicolumn{2}{|l|}{ Peak VO2 (mL/Kg/min) } & $\begin{array}{l}\text { No mortality ( } 30 \\
\text { days) }\end{array}$ & Mortality (30 days) & $1.03(0.77-1.37)$ & \\
\hline Junejo 2014 & $\begin{array}{l}\text { Pancreatic } \\
\text { (64) }\end{array}$ & \multicolumn{2}{|l|}{ Peak $\mathrm{VO}_{2}(\mathrm{~mL} / \mathrm{Kg} / \mathrm{min})$} & $\begin{array}{l}\text { No mortality (in- } \\
\text { hospital) }\end{array}$ & $\begin{array}{l}\text { Mortality (in- } \\
\text { hospital) }\end{array}$ & $1.32(0.91-1.93)$ & \\
\hline West 2014 & Rectal (46) & $\begin{array}{l}\text { Peak } \mathrm{VO}_{2} \geq 10.6 \\
\quad(\mathrm{~mL} / \mathrm{Kg} / \mathrm{min})\end{array}$ & $\begin{array}{l}\text { Peak } \mathrm{VO}_{2}<10.6 \\
(\mathrm{~mL} / \mathrm{Kg} / \mathrm{min})\end{array}$ & Minor complication & Major complication & $0.60(0.13-2.49)$ & \\
\hline $\begin{array}{l}\text { McSorley } \\
2018\end{array}$ & $\begin{array}{l}\text { Colorectal } \\
\text { (38) }\end{array}$ & $\begin{array}{l}\text { Peak } \mathrm{VO}_{2}>19(\mathrm{~mL} / \\
\mathrm{Kg} / \mathrm{min})\end{array}$ & $\begin{array}{l}\text { Peak } \mathrm{VO}_{2}<19(\mathrm{~mL} / \\
\mathrm{Kg} / \mathrm{min})\end{array}$ & Minor complication & Major complication & $1.00(0.08-11.67)$ & \\
\hline Dunne 2014 & $\begin{array}{l}\text { Liver } \\
\quad(194)\end{array}$ & \multicolumn{2}{|l|}{ Peak $\mathrm{VO}_{2}(\mathrm{~mL} / \mathrm{Kg} / \mathrm{min})$} & Minor complication & Major complication & $1.04(0.97-1.11)$ & \\
\hline Huang 2016 & Mixed (26) & \multicolumn{2}{|l|}{ Peak $\mathrm{VO}_{2}(\mathrm{~mL} / \mathrm{Kg} / \mathrm{min})$} & Minor complication & Major complication & $0.72(0.17-2.21)$ & \\
\hline West 2014 & Rectal (95) & $\begin{array}{l}\text { Peak } \mathrm{VO}_{2} \geq 10.6 \\
\quad(\mathrm{~mL} / \mathrm{Kg} / \mathrm{min})\end{array}$ & $\begin{array}{c}\text { Peak } \mathrm{VO}_{2}<10.6 \\
\quad(\mathrm{~mL} / \mathrm{Kg} / \mathrm{min})\end{array}$ & No infection & Infection & $0.10(0.03-0.26)$ & \\
\hline West 2014 & Rectal (95) & $\begin{array}{l}\text { Peak } \mathrm{VO}_{2} \geq 10.6 \\
\quad(\mathrm{~mL} / \mathrm{Kg} / \mathrm{min})\end{array}$ & $\begin{array}{l}\text { Peak } \mathrm{VO}_{2}<10.6 \\
(\mathrm{~mL} / \mathrm{Kg} / \mathrm{min})\end{array}$ & No wound dehiscence & Wound dehiscence & $0.10(0.00-1.16)$ & \\
\hline West 2014 & Rectal (95) & $\begin{array}{l}\text { Peak } \mathrm{VO}_{2} \geq 10.6 \\
\quad(\mathrm{~mL} / \mathrm{Kg} / \mathrm{min})\end{array}$ & $\begin{array}{c}\text { Peak } \mathrm{VO}_{2}<10.6 \\
(\mathrm{~mL} / \mathrm{Kg} / \mathrm{min})\end{array}$ & No renal complication & Renal complication & $0.20(0.04-1.07)$ & \\
\hline West 2014 & Rectal (95) & $\begin{array}{l}\text { Peak } \mathrm{VO}_{2} \geq 10.6 \\
\quad(\mathrm{~mL} / \mathrm{Kg} / \mathrm{min})\end{array}$ & $\begin{array}{l}\text { Peak } \mathrm{VO}_{2}<10.6 \\
\quad(\mathrm{~mL} / \mathrm{Kg} / \mathrm{min})\end{array}$ & $\begin{array}{l}\text { No gastrointestinal } \\
\text { complication }\end{array}$ & $\begin{array}{r}\text { Gastrointestinal } \\
\text { complication }\end{array}$ & $0.30(0.09-0.80)$ & \\
\hline West 2014 & Rectal (95) & $\begin{array}{l}\text { Peak } \mathrm{VO}_{2} \geq 10.6 \\
\quad(\mathrm{~mL} / \mathrm{Kg} / \mathrm{min})\end{array}$ & $\begin{array}{c}\text { Peak } \mathrm{VO}_{2}<10.6 \\
\quad(\mathrm{~mL} / \mathrm{Kg} / \mathrm{min})\end{array}$ & $\begin{array}{c}\text { No neurological } \\
\text { complication }\end{array}$ & $\begin{array}{l}\text { Neurological } \\
\text { complication }\end{array}$ & $0.70(0.01-35.72)$ & \\
\hline West 2014 & Rectal (95) & $\begin{array}{l}\text { Peak } \mathrm{VO}_{2} \geq 10.6 \\
\quad(\mathrm{~mL} / \mathrm{Kg} / \mathrm{min})\end{array}$ & $\begin{array}{l}\text { Peak } \mathrm{VO}_{2}<10.6 \\
(\mathrm{~mL} / \mathrm{Kg} / \mathrm{min})\end{array}$ & $\begin{array}{l}\text { No hematological } \\
\text { complication }\end{array}$ & $\begin{array}{l}\text { Hematological } \\
\text { complication }\end{array}$ & $0.70(0.13-3.56)$ & \\
\hline West 2014 & Rectal (95) & $\begin{array}{l}\text { Peak } \mathrm{VO}_{2} \geq 10.6 \\
\quad(\mathrm{~mL} / \mathrm{Kg} / \mathrm{min})\end{array}$ & $\begin{array}{c}\text { Peak } \mathrm{VO}_{2}<10.6 \\
(\mathrm{~mL} / \mathrm{Kg} / \mathrm{min})\end{array}$ & No pain & Pain & $2.9(0.31-27.21)$ & \\
\hline Bayram 2007 & Lung (55) & $\begin{array}{c}\text { Peak } \mathrm{VO}_{2}>15(\mathrm{~mL} / \\
\mathrm{Kg} / \mathrm{min})\end{array}$ & $\begin{array}{c}\text { Peak } \mathrm{VO}_{2}<15(\mathrm{~mL} / \\
\mathrm{Kg} / \mathrm{min})\end{array}$ & No Pneumonia & Pneumonia & $0.20(0.01-3.30)$ & \\
\hline Bayram 2007 & Lung (55) & $\begin{array}{l}\text { Peak } \mathrm{VO}_{2}>15(\mathrm{~mL} / \\
\mathrm{Kg} / \mathrm{min})\end{array}$ & $\begin{array}{l}\text { Peak } \mathrm{VO}_{2}<15(\mathrm{~mL} / \\
\mathrm{Kg} / \mathrm{min})\end{array}$ & No atelectasis & Atelectasis & $0.10(0.0-1.31)$ & \\
\hline Bayram 2007 & Lung (55) & $\begin{array}{c}\text { Peak } \mathrm{VO}_{2}>15(\mathrm{~mL} / \\
\mathrm{Kg} / \mathrm{min})\end{array}$ & $\begin{array}{l}\text { Peak } \mathrm{VO}_{2}<15(\mathrm{~mL} / \\
\mathrm{Kg} / \mathrm{min})\end{array}$ & $\begin{array}{l}\text { No bronchopleural } \\
\text { fistula }\end{array}$ & $\begin{array}{l}\text { Bronchopleural } \\
\text { fistula }\end{array}$ & $0.20(0.01-5.70)$ & \\
\hline
\end{tabular}


TABLE 3 continued

\begin{tabular}{|c|c|c|c|c|c|c|c|}
\hline \multirow[t]{2}{*}{ Author, year } & \multirow{2}{*}{$\begin{array}{l}\text { Cancer type } \\
\text { (N) }\end{array}$} & \multicolumn{2}{|c|}{ Preoperative CPET threshold } & \multicolumn{2}{|c|}{ Postoperative complication } & \multirow{2}{*}{$\begin{array}{l}\text { Estimates, odds ratio ( } 95 \% \\
\text { confidence intervals) }\end{array}$} & \multirow[t]{2}{*}{ Summary } \\
\hline & & Favorable & Unfavorable & Favorable outcome & $\begin{array}{l}\text { Unfavorable } \\
\text { outcome }\end{array}$ & & \\
\hline Bayram 2007 & Lung (55) & $\begin{array}{l}\text { Peak } \mathrm{VO}_{2}>15 \\
\quad(\mathrm{~mL} / \mathrm{Kg} / \mathrm{min})\end{array}$ & $\begin{array}{l}\text { Peak } \mathrm{VO}_{2}<15 \\
\quad(\mathrm{~mL} / \mathrm{Kg} / \mathrm{min})\end{array}$ & No prolonged air leak & Prolonged air leak & $0.50(0.15-1.46)$ & \\
\hline Bayram 2007 & Lung (55) & $\begin{array}{l}\text { Peak } \mathrm{VO}_{2}>15 \\
\quad(\mathrm{~mL} / \mathrm{Kg} / \mathrm{min})\end{array}$ & $\begin{array}{l}\text { Peak } \mathrm{VO}_{2}<15 \\
\quad(\mathrm{~mL} / \mathrm{Kg} / \mathrm{min})\end{array}$ & No arrhythmia & Arrhytmia & $2.2(0.18-25.32)$ & \\
\hline Ausania 2012 & $\begin{array}{c}\text { Pancreas } \\
(124)\end{array}$ & $\begin{array}{l}\mathrm{AT} \geq 10.1(\mathrm{~mL} / \mathrm{Kg} / \\
\mathrm{min})\end{array}$ & $\begin{array}{l}\mathrm{AT}<10.1(\mathrm{~mL} / \mathrm{Kg} / \\
\mathrm{min})\end{array}$ & No complication & Complication & $0.27(0.10-0.75)$ & \\
\hline Chan 2016 & $\begin{array}{l}\text { Colorectal } \\
\quad(48)\end{array}$ & $\mathrm{AT}(\mathrm{mL} / \mathrm{Kg} / \mathrm{min})$ & & No complication & Complication & NR & \\
\hline Junejo 2014 & $\begin{array}{l}\text { Pancreas } \\
\text { (64) }\end{array}$ & $\mathrm{AT}(\mathrm{mL} / \mathrm{Kg} / \mathrm{min})$ & & No complication & Complication & $1.07(0.83-1.39)$ & \\
\hline Sinclair 2017 & $\begin{array}{l}\text { Esophagus } \\
(240)\end{array}$ & $\mathrm{AT}(\mathrm{mL} / \mathrm{Kg} / \mathrm{min})$ & & No complication & Complication & $0.95(0.90-1.01)$ & \\
\hline $\begin{array}{l}\text { Chandrabalan } \\
2013\end{array}$ & $\begin{array}{l}\text { Pancreatic } \\
\quad(100)\end{array}$ & $\begin{array}{l}\mathrm{AT} \geq 10(\mathrm{~mL} / \mathrm{Kg} / \\
\min )\end{array}$ & $\begin{array}{l}\mathrm{AT}<10(\mathrm{~mL} / \mathrm{Kg} / \\
\mathrm{min})\end{array}$ & No mortality (30 days) & Mortality (30 days) & $1.30(0.28-6.16)$ & \\
\hline Wilson 2010 & $\begin{array}{l}\text { Mixed } \\
(847)\end{array}$ & $\begin{array}{l}\mathrm{AT}>10.9(\mathrm{ml} / \mathrm{kg} / \\
\quad \min )\end{array}$ & $\begin{array}{l}\mathrm{AT} \leq 10.9(\mathrm{ml} / \mathrm{kg} / \\
\min )\end{array}$ & No mortality (90 days) & Mortality (90 days) & $0.14(0.03-0.62)$ & \\
\hline Bowles 2008 & $\begin{array}{l}\text { Colorectal } \\
\text { (121) }\end{array}$ & $\begin{array}{l}\mathrm{AT}>11(\mathrm{ml} / \mathrm{kg} / \\
\min )\end{array}$ & $\begin{array}{l}\mathrm{AT} \leq 11(\mathrm{ml} / \mathrm{kg} / \\
\min )\end{array}$ & No mortality (NR) & Mortality (NR) & $2.56(0.29-22.73)$ & \\
\hline Junejo 2014 & $\begin{array}{l}\text { Pancreas } \\
\text { (64) }\end{array}$ & $\mathrm{AT}(\mathrm{mL} / \mathrm{Kg} / \mathrm{min})$ & & $\begin{array}{l}\text { No mortality (in- } \\
\text { hospital) }\end{array}$ & $\begin{array}{l}\text { Mortality (in- } \\
\text { hospital) }\end{array}$ & $0.90(0.52-1.53)$ & \\
\hline Ausania 2012 & $\begin{array}{c}\text { Pancreas } \\
(124)\end{array}$ & $\begin{array}{l}\mathrm{AT} \geq 10.1(\mathrm{~mL} / \mathrm{Kg} / \\
\min )\end{array}$ & $\begin{array}{l}\mathrm{AT}<10.1(\mathrm{~mL} / \mathrm{Kg} / \\
\min )\end{array}$ & $\begin{array}{l}\text { No mortality (in- } \\
\text { hospital) }\end{array}$ & $\begin{array}{l}\text { Mortality (in- } \\
\text { hospital) }\end{array}$ & $0.76(0.08-7.18)$ & \\
\hline Junejo 2014 & $\begin{array}{l}\text { Pancreas } \\
\text { (64) }\end{array}$ & $\mathrm{AT}(\mathrm{mL} / \mathrm{Kg} / \mathrm{min})$ & & No mortality (30 days) & Mortality (30 days) & $1.23(0.72-2.11)$ & \\
\hline Mann 2020 & $\begin{array}{c}\text { Colorectal } \\
\quad(1205)\end{array}$ & $\begin{array}{l}\mathrm{AT} \geq 11(\mathrm{~mL} / \mathrm{Kg} / \\
\min )\end{array}$ & $\begin{array}{l}\mathrm{AT}<11(\mathrm{~mL} / \mathrm{Kg} / \\
\min )\end{array}$ & No mortality (30 days) & Mortality (30 days) & $0.70(0.32-1.51)$ & \\
\hline Moyes 2013 & $\begin{array}{l}\text { Mixed } \\
\quad(103)\end{array}$ & $\begin{array}{l}\mathrm{AT} \geq 9(\mathrm{~mL} / \mathrm{Kg} / \\
\min )\end{array}$ & $\begin{array}{l}\mathrm{AT}<9(\mathrm{~mL} / \mathrm{Kg} / \\
\mathrm{min})\end{array}$ & $\begin{array}{l}\text { No cardiopulmonary } \\
\text { complication }\end{array}$ & $\begin{array}{l}\text { Cardiopulmonary } \\
\text { complication }\end{array}$ & $0.40(0.16-1.07)$ & \\
\hline Moyes 2013 & $\begin{array}{l}\text { Mixed } \\
\quad(103)\end{array}$ & $\begin{array}{c}\mathrm{AT} \geq 11(\mathrm{~mL} / \mathrm{Kg} / \\
\min )\end{array}$ & $\begin{array}{l}\mathrm{AT}<11(\mathrm{~mL} / \mathrm{Kg} / \\
\min )\end{array}$ & $\begin{array}{l}\text { No cardiopulmonary } \\
\text { complication }\end{array}$ & $\begin{array}{l}\text { Cardiopulmonary } \\
\text { complication }\end{array}$ & $0.50(0.18-1.12)$ & \\
\hline Forshaw 2008 & $\begin{array}{l}\text { Esophagus } \\
\text { (75) }\end{array}$ & $\begin{array}{l}\mathrm{AT}>11(\mathrm{~mL} / \mathrm{Kg} / \\
\min )\end{array}$ & $\begin{array}{l}\mathrm{AT}<11(\mathrm{~mL} / \mathrm{Kg} / \\
\min )\end{array}$ & $\begin{array}{l}\text { No cardiopulmonary } \\
\text { complication }\end{array}$ & $\begin{array}{l}\text { Cardiopulmonary } \\
\text { complication }\end{array}$ & $0.40(0.12-1.44)$ & \\
\hline Sinclair 2017 & $\begin{array}{l}\text { Esophagus } \\
\text { (240) }\end{array}$ & $\mathrm{AT}(\mathrm{mL} / \mathrm{Kg} / \mathrm{min})$ & & $\begin{array}{l}\text { No cardiopulmonary } \\
\text { complication }\end{array}$ & $\begin{array}{l}\text { Cardiopulmonary } \\
\text { complication }\end{array}$ & $0.89(0.84-0.95)$ & \\
\hline
\end{tabular}


TABLE 3 continued

\begin{tabular}{|c|c|c|c|c|c|c|c|}
\hline \multirow[t]{2}{*}{ Author, year } & \multirow{2}{*}{$\begin{array}{l}\text { Cancer } \\
\text { type }(\mathrm{N})\end{array}$} & \multicolumn{2}{|c|}{ Preoperative CPET threshold } & \multicolumn{2}{|l|}{ Postoperative complication } & \multirow{2}{*}{$\begin{array}{l}\text { Estimates, odds ratio (95\% } \\
\text { confidence intervals) }\end{array}$} & \multirow[t]{2}{*}{ Summary } \\
\hline & & Favorable & Unfavorable & Favorable outcome & Unfavorable outcome & & \\
\hline Junejo 2014 & $\begin{array}{l}\text { Pancreas } \\
\text { (64) }\end{array}$ & $\mathrm{AT}(\mathrm{mL} / \mathrm{Kg} / \mathrm{min})$ & & $\begin{array}{l}\text { No cardiopulmonary } \\
\text { complication }\end{array}$ & $\begin{array}{l}\text { Cardiopulmonary } \\
\text { complication }\end{array}$ & $1.05(0.82-1.34)$ & \\
\hline Forshaw 2008 & $\begin{array}{l}\text { Esophagus } \\
\quad(75)\end{array}$ & $\begin{array}{c}\mathrm{AT}>11(\mathrm{~mL} / \\
\mathrm{Kg} / \mathrm{min})\end{array}$ & $\begin{array}{c}\mathrm{AT}<11(\mathrm{~mL} / \\
\mathrm{Kg} / \mathrm{min})\end{array}$ & $\begin{array}{l}\text { No Noncardiopulmonary } \\
\text { complication }\end{array}$ & $\begin{array}{l}\text { Noncardiopulmonary } \\
\text { complication }\end{array}$ & $1.60(0.31-7.94)$ & \\
\hline Lamb 2016 & $\begin{array}{c}\text { Bladder } \\
\text { (82) }\end{array}$ & $\mathrm{AT} \geq 11(\mathrm{NR})$ & AT $<11$ (NR) & $\begin{array}{l}\text { Minor complication }(\mathrm{CD} \\
<3)\end{array}$ & $\begin{array}{l}\text { Major complication (CD } \\
\text { 3-5) }\end{array}$ & $1.10(0.30-3.85)$ & \\
\hline Bowles 2008 & $\begin{array}{l}\text { Colorectal } \\
\text { (121) }\end{array}$ & $\mathrm{AT} \geq 11(\mathrm{NR})$ & $\mathrm{AT}<11(\mathrm{NR})$ & $\begin{array}{l}\text { Minor complication }(\mathrm{CD} \\
\quad<3)\end{array}$ & $\begin{array}{l}\text { Major complication }(\mathrm{CD} \\
\quad 3-5)\end{array}$ & $1.45(0.55-3.79)$ & \\
\hline Lamb 2016 & $\begin{array}{c}\text { Bladder } \\
\quad(82)\end{array}$ & $\mathrm{AT} \geq 11(\mathrm{NR})$ & $\mathrm{AT}<11(\mathrm{NR})$ & $\begin{array}{l}\text { Minor complication }(\mathrm{CD} \\
\quad<3)\end{array}$ & $\begin{array}{l}\text { Major complication (CD } \\
\quad 3-5)\end{array}$ & $0.30(0.04-2.46)$ & \\
\hline $\begin{array}{l}\text { Chandrabalan } \\
2013\end{array}$ & $\begin{array}{c}\text { Pancreas } \\
(100)\end{array}$ & $\begin{array}{c}\mathrm{AT} \geq 10(\mathrm{~mL} / \\
\mathrm{Kg} / \mathrm{min})\end{array}$ & $\begin{array}{c}\mathrm{AT}<10(\mathrm{~mL} / \\
\mathrm{Kg} / \mathrm{min})\end{array}$ & $\begin{array}{l}\text { Minor cardiac } \\
\text { complications }(C D<3)\end{array}$ & $\begin{array}{l}\text { Major cardiac } \\
\quad \text { complications (CD 3-5) }\end{array}$ & $0.50(0.02-14.5)$ & \\
\hline $\begin{array}{l}\text { Chandrabalan } \\
2013\end{array}$ & $\begin{array}{c}\text { Pancreas } \\
(100)\end{array}$ & $\begin{array}{c}\mathrm{AT} \geq 10(\mathrm{~mL} / \\
\mathrm{Kg} / \mathrm{min})\end{array}$ & $\begin{array}{c}\mathrm{AT}<10(\mathrm{~mL} / \\
\mathrm{Kg} / \mathrm{min})\end{array}$ & $\begin{array}{l}\text { Minor respiratory } \\
\text { complications }(C D<3)\end{array}$ & $\begin{array}{l}\text { Major respiratory } \\
\text { complications (CD 3-5) }\end{array}$ & $0.70(0.15-3.32)$ & \\
\hline $\begin{array}{l}\text { Chandrabalan } \\
2013\end{array}$ & $\begin{array}{c}\text { Pancreas } \\
(100)\end{array}$ & $\begin{array}{c}\mathrm{AT} \geq 10(\mathrm{~mL} / \\
\mathrm{Kg} / \mathrm{min})\end{array}$ & $\begin{array}{c}\mathrm{AT}<10(\mathrm{~mL} / \\
\mathrm{Kg} / \mathrm{min})\end{array}$ & $\begin{array}{l}\text { Minor intra-abdominal } \\
\text { abscess }(\mathrm{CD}<3)\end{array}$ & $\begin{array}{l}\text { Major intra-abdominal } \\
\text { abscess (CD 3-5) }\end{array}$ & $0.30(0.09-1.00)$ & \\
\hline $\begin{array}{l}\text { Chandrabalan } \\
2013\end{array}$ & $\begin{array}{l}\text { Pancreas } \\
\quad(98)\end{array}$ & $\begin{array}{c}\mathrm{AT} \geq 10(\mathrm{~mL} / \\
\mathrm{Kg} / \mathrm{min})\end{array}$ & $\begin{array}{c}\mathrm{AT}<10(\mathrm{~mL} / \\
\mathrm{Kg} / \mathrm{min})\end{array}$ & No pancreatic fistula & Pancreatic fistula & $0.30(0.13-0.91)$ & \\
\hline $\begin{array}{l}\text { Chandrabalan } \\
2013\end{array}$ & $\begin{array}{c}\text { Pancreas } \\
(100)\end{array}$ & $\begin{array}{c}\mathrm{AT} \geq 10(\mathrm{~mL} / \\
\mathrm{Kg} / \mathrm{min})\end{array}$ & $\begin{array}{c}\mathrm{AT}<10(\mathrm{~mL} / \\
\mathrm{Kg} / \mathrm{min})\end{array}$ & No hemorrhage & Hemorrhage & $1.70(0.58-5.25)$ & \\
\hline Ausania 2012 & $\begin{array}{c}\text { Pancreas } \\
(124)\end{array}$ & $\begin{array}{c}\mathrm{AT} \geq 10.1(\mathrm{~mL} / \\
\mathrm{Kg} / \mathrm{min})\end{array}$ & $\begin{array}{c}\mathrm{AT}<10.1(\mathrm{~mL} / \\
\mathrm{Kg} / \mathrm{min})\end{array}$ & $\begin{array}{l}\text { No cardiorespiratory } \\
\text { complications }\end{array}$ & $\begin{array}{r}\text { Cardiorespiratory } \\
\text { complications }\end{array}$ & $0.30(0.08-1.52)$ & \\
\hline Whibley 2018 & Mixed (81) & $\mathrm{AT} \geq 11(\mathrm{NR})$ & $\mathrm{AT}<11(\mathrm{NR})$ & $\begin{array}{l}\text { No respiratory } \\
\text { complications }\end{array}$ & Respiratory complications & NR & \\
\hline Forshaw 2008 & $\begin{array}{l}\text { Esophagus } \\
\quad(75)\end{array}$ & $\begin{array}{c}\mathrm{AT}>11(\mathrm{~mL} / \\
\mathrm{Kg} / \mathrm{min})\end{array}$ & $\begin{array}{c}\mathrm{AT}<11(\mathrm{~mL} / \\
\mathrm{Kg} / \mathrm{min})\end{array}$ & $\begin{array}{l}\text { No unplanned ITU } \\
\text { admission }\end{array}$ & Unplanned ITU admission & $0.60(0.13-2.46)$ & \\
\hline Junejo 2014 & $\begin{array}{c}\text { Pancreas } \\
\quad(64)\end{array}$ & $\mathrm{V}_{\mathrm{E}} / \mathrm{V}_{\mathrm{CO} 2}$ & & No complication & Complication & $0.97(0.89-1.07)$ & \\
\hline Dunne 2014 & Liver (194) & $\mathrm{V}_{\mathrm{E}} / \mathrm{V}_{\mathrm{CO} 2}$ & & No complication & Complication & $0.98(0.93-1.04)$ & \\
\hline Sinclair 2017 & $\begin{array}{l}\text { Esophagus } \\
\quad(240)\end{array}$ & $\mathrm{V}_{\mathrm{E}} / \mathrm{V}_{\mathrm{CO} 2}$ & & No complication & Complication & $0.90(0.84-0.96)$ & \\
\hline Junejo 2014 & $\begin{array}{c}\text { Pancreas } \\
\quad(64)\end{array}$ & $\mathrm{V}_{\mathrm{E}} / \mathrm{V}_{\mathrm{CO} 2}$ & & No mortality (in-hospital) & Mortality (in-hospital) & $0.79(0.66-0.95)$ & \\
\hline Wilson 2010 & $\begin{array}{l}\text { Mixed } \\
(847)\end{array}$ & $\mathrm{V}_{\mathrm{E}} / \mathrm{V}_{\mathrm{CO} 2}<34$ & $\mathrm{~V}_{\mathrm{E}} / \mathrm{V}_{\mathrm{CO} 2} \geq 34$ & No mortality (in-hospital) & Mortality (in-hospital) & $0.20(0.06-0.74)$ & \\
\hline
\end{tabular}


TABLE 3 continued

\begin{tabular}{|c|c|c|c|c|c|c|c|}
\hline \multirow[t]{2}{*}{ Author, year } & \multirow[t]{2}{*}{$\begin{array}{l}\text { Cancer type } \\
\text { (N) }\end{array}$} & \multicolumn{2}{|c|}{$\begin{array}{l}\text { Preoperative CPET } \\
\text { threshold }\end{array}$} & \multicolumn{2}{|l|}{ Postoperative complication } & \multirow[t]{2}{*}{$\begin{array}{l}\text { Estimates, odds ratio ( } 95 \% \text { confidence } \\
\text { intervals) }\end{array}$} & \multirow[t]{2}{*}{ Summary } \\
\hline & & Favorable & Unfavorable & Favorable outcome & Unfavorable outcome & & \\
\hline Junejo 2014 & Pancreas (64) & $\mathrm{V}_{\mathrm{E}} / \mathrm{V}_{\mathrm{CO} 2}$ & & No mortality (30 days) & Mortality (30 days) & $0.74(0.56-0.97)$ & \\
\hline Mann 2020 & $\begin{array}{c}\text { Colorectal } \\
\text { (1193) }\end{array}$ & $\begin{array}{c}\mathrm{V}_{\mathrm{E}} / \mathrm{V}_{\mathrm{CO} 2} \\
\leq 34\end{array}$ & $\begin{array}{c}\mathrm{V}_{\mathrm{E}} / \mathrm{V}_{\mathrm{CO} 2} \\
>34\end{array}$ & No mortality (30 days) & Mortality (30 days) & $0.30(0.12-0.68)$ & \\
\hline $\begin{array}{c}\text { Miyazaki } \\
2018\end{array}$ & Lung (172) & $\begin{array}{c}\mathrm{V}_{\mathrm{E}} / \mathrm{V}_{\mathrm{CO} 2} \\
\quad<40\end{array}$ & $\begin{array}{c}\mathrm{V}_{\mathrm{E}} / \mathrm{V}_{\mathrm{CO} 2} \\
>40\end{array}$ & No mortality (90 days) & Mortality (90 days) & $0.30(0.09-0.86)$ & \\
\hline $\begin{array}{c}\text { Miyazaki } \\
2018\end{array}$ & Lung (172) & $\begin{aligned} \mathrm{V}_{\mathrm{E}} / \mathrm{V}_{\mathrm{CO} 2} \\
\quad<40\end{aligned}$ & $\begin{array}{c}\mathrm{V}_{\mathrm{E}} / \mathrm{V}_{\mathrm{CO} 2} \\
>40\end{array}$ & $\begin{array}{l}\text { No cardiopulmonary } \\
\text { complication }\end{array}$ & $\begin{array}{l}\text { Cardiopulmonary } \\
\text { complication }\end{array}$ & $0.60(0.32-1.26)$ & \\
\hline Junejo 2014 & Pancreas (64) & $\mathrm{V}_{\mathrm{E}} / \mathrm{V}_{\mathrm{CO} 2}$ & & $\begin{array}{l}\text { No cardiopulmonary } \\
\text { complication }\end{array}$ & $\begin{array}{l}\text { Cardiopulmonary } \\
\text { complication }\end{array}$ & $0.98(0.90-1.07)$ & \\
\hline Sinclair 2017 & $\begin{array}{l}\text { Esophagus } \\
\text { (240) }\end{array}$ & $\mathrm{V}_{\mathrm{E}} / \mathrm{V}_{\mathrm{CO} 2}$ & & $\begin{array}{l}\text { No cardiopulmonary } \\
\text { complication }\end{array}$ & $\begin{array}{l}\text { Cardiopulmonary } \\
\text { complication }\end{array}$ & $0.91(0.85-0.96)$ & \\
\hline Mann 2020 & $\begin{array}{c}\text { Colorectal } \\
\text { (1193) }\end{array}$ & $\begin{array}{l}\mathrm{V}_{\mathrm{E}} / \mathrm{V}_{\mathrm{CO} 2} \\
\quad \leq 34\end{array}$ & $\begin{array}{c}\mathrm{V}_{\mathrm{E}} / \mathrm{V}_{\mathrm{CO} 2} \\
>34\end{array}$ & No unplanned critical care & Unplanned critical care & $0.80(0.53-1.30)$ & \\
\hline Dunne 2014 & Liver (194) & $\mathrm{V}_{\mathrm{E}} / \mathrm{V}_{\mathrm{CO} 2}$ & & $\begin{array}{l}\text { Minor complication }(\mathrm{CD} \\
\quad<3)\end{array}$ & $\begin{array}{l}\text { Major Complication (CD } \\
>3 \text { ) }\end{array}$ & $0.99(0.93-1.05)$ & \\
\hline
\end{tabular}

Significant association between favorable preoperative cardiopulmonary exercise test (CPET) variables and absence of postoperative complications

No significant association between preoperative CPET variables and postoperative compilations

Significant association between unfavourable preoperative CPET variables and absence of postoperative complications

Peak $\mathrm{VO}_{2}=$ peak oxygen uptake; $\mathrm{AT}=$ anaerobic threshold; $\mathrm{V}_{\mathrm{E}} / \mathrm{V}_{\mathrm{CO} 2}=$ ventilatory equivalent for carbon dioxide

in most of the previous systematic reviews. This has prevented the pooling of a larger number of studies and a stronger conclusion across the identified reviews.

\section{Meaning of the Study}

Despite the advances in the medical field and surgical approaches, postoperative complications following cancer surgery remain high, increasing the length of hospital stay and subsequently hospital costs. Therefore, identifying preoperative factors that accurately predict adverse postoperative outcomes would be of great benefit to inform potential optimization strategies, improve the processes of shared decision making, and informed consent in patients presenting for major cancer surgery. In a mixed group of cancer patients undergoing surgery, our systematic review and meta-analysis found that superior CPET values were associated with improved postoperative outcomes. Furthermore, results for individual studies, not included in the meta-analysis, also provided some positive trends. These also trends have been reported in other systematic reviews. $^{5,7,8}$ Therefore, the assessment of functional capacity in the preoperative period should be used in conjunction with other clinical assessments to support clinicians, patients, and payers on optimization strategies and treatment decision making. This has the potential to provide the best possible outcome for patients and reduce the economic burden.

\section{Unanswered Questions and Future Research}

One of the goals of this study was to explore the association between preoperative CPET values and postoperative quality of life outcomes. Unfortunately, none of the included literature assessed this potential association. Future prospective cohort studies should include quality of life as one of the postoperative outcomes of interest. Our review not only focused on continuous measures of preoperative CPET, but also extracted dichotomous outcomes, or potential CPET cutoff points. This information was 
TABLE 4 Association between preoperative cardiopulmonary exercise test variables and postoperative complications

\begin{tabular}{|c|c|c|c|c|c|c|c|}
\hline \multirow[t]{2}{*}{ Author year } & \multirow{2}{*}{$\begin{array}{l}\text { Cancer type } \\
(\mathrm{N})\end{array}$} & \multirow{2}{*}{$\begin{array}{l}\text { CPET } \\
\text { variable }\end{array}$} & \multirow[t]{2}{*}{ Outcome } & \multicolumn{2}{|c|}{ Postoperative complication } & \multirow[t]{2}{*}{ Estimates } & \multirow[t]{2}{*}{ Summary } \\
\hline & & & & Absent & Present & & \\
\hline Lamb 2016 & $\begin{array}{l}\text { Bladder } \\
\quad(82)\end{array}$ & $\begin{array}{l}\text { Peak } \mathrm{VO}_{2} \\
\quad(\mathrm{ml} / \mathrm{kg} / \\
\mathrm{min})\end{array}$ & $\begin{array}{l}\text { Major } \\
\text { complication } \\
\text { (CD 3-5) }\end{array}$ & Median: 17.00 & Median: 15.00 & NR & $\Theta$ \\
\hline Forshaw 2008 & $\begin{array}{l}\text { Esophageal } \\
\text { (78) }\end{array}$ & $\begin{array}{l}\text { Peak } \mathrm{VO}_{2} \\
\quad(\mathrm{ml} / \mathrm{kg} / \\
\mathrm{min})\end{array}$ & $\begin{array}{l}\text { Unplanned ICU } \\
\text { admission }\end{array}$ & $\begin{array}{c}\text { Mean (SD): } \\
20.80 \\
(5.00)\end{array}$ & $\begin{array}{c}\text { Mean (SD): } \\
18.90 \\
(5.10)\end{array}$ & $\begin{array}{c}\text { Mean difference }(95 \% \mathrm{CI}) \text { : } \\
1.90(-1.10 \text { to } 4.90)\end{array}$ & $\Theta$ \\
\hline Brunelli 2009 & Lung (204) & $\begin{array}{l}\text { Peak } \mathrm{VO}_{2} \\
\quad(\mathrm{ml} / \mathrm{kg} / \\
\mathrm{min})\end{array}$ & $\begin{array}{l}\text { Cardiac } \\
\quad \text { complication }\end{array}$ & $\begin{array}{c}\text { Mean (SD): } \\
16.00 \\
(3.80)\end{array}$ & $\begin{array}{c}\text { Mean (SD): } \\
15.00 \\
(3.70)\end{array}$ & $\begin{array}{c}\text { Mean difference }(95 \% \mathrm{CI}) \text { : } \\
1.00(-0.50 \text { to } 2.50)\end{array}$ & $\Theta$ \\
\hline $\begin{array}{l}\text { Bechard and } \\
\text { Wetstein } \\
1987\end{array}$ & Lung (29) & AT (L/Min) & Any complication & $\begin{array}{l}\text { Mean (SD): } \\
0.93(0.20)\end{array}$ & $\begin{array}{l}\text { Mean (SD): } \\
0.61(0.10)\end{array}$ & $\begin{array}{l}\text { Mean difference }(95 \% \mathrm{CI}) \text { : } \\
0.32(0.10 \text { to } 0.60)\end{array}$ & $\oplus$ \\
\hline Lamb 2016 & $\begin{array}{l}\text { Bladder } \\
\quad(82)\end{array}$ & $\begin{array}{l}\mathrm{AT}(\mathrm{ml} / \mathrm{kg} / \\
\mathrm{min})\end{array}$ & $\begin{array}{l}\text { Major } \\
\text { complication } \\
\text { (CD 3-5) }\end{array}$ & Median: 10.00 & Median: 11.00 & NR & $\Theta$ \\
\hline Brunelli 2009 & Lung (204) & $\begin{array}{l}\mathrm{AT}(\mathrm{ml} / \mathrm{kg} / \\
\min )\end{array}$ & $\begin{array}{l}\text { Pulmonary } \\
\text { complication }\end{array}$ & $\begin{array}{c}\text { Mean (SD): } \\
10.10 \\
(3.80)\end{array}$ & $\begin{array}{l}\text { Mean (SD): } \\
\quad 9.20(1.90)\end{array}$ & $\begin{array}{c}\text { Mean difference }(95 \% \mathrm{CI}) \text { : } \\
0.90(-0.50 \text { to } 2.30)\end{array}$ & $\Theta$ \\
\hline Brunelli 2009 & Lung (204) & $\begin{array}{l}\mathrm{AT}(\mathrm{ml} / \mathrm{kg} / \\
\min )\end{array}$ & $\begin{array}{l}\text { Cardiac } \\
\quad \text { complication }\end{array}$ & $\begin{array}{c}\text { Mean (SD): } \\
10.00 \\
(3.80)\end{array}$ & $\begin{array}{l}\text { Mean (SD): } \\
9.90(1.60)\end{array}$ & $\begin{array}{c}\text { Mean difference }(95 \% \mathrm{CI}) \text { : } \\
0.10(-1.40 \text { to } 1.60)\end{array}$ & $\Theta$ \\
\hline Forshaw 2008 & $\begin{array}{l}\text { Esophageal } \\
\text { (78) }\end{array}$ & $\begin{array}{l}\mathrm{AT}(\mathrm{ml} / \mathrm{kg} / \\
\mathrm{min})\end{array}$ & $\begin{array}{l}\text { Unplanned ICU } \\
\text { admission }\end{array}$ & $\begin{array}{c}\text { Mean (SD): } \\
14.20 \\
(2.80)\end{array}$ & $\begin{array}{c}\text { Mean (SD): } \\
12.60 \\
(3.20)\end{array}$ & $\begin{array}{c}\text { Mean difference }(95 \% \mathrm{CI}) \text { : } \\
1.60(-0.10 \text { to } 3.30)\end{array}$ & $\Theta$ \\
\hline Lamb 2016 & $\begin{array}{l}\text { Bladder } \\
\quad(45)\end{array}$ & $\mathrm{V}_{\mathrm{E}} / \mathrm{V}_{\mathrm{CO} 2}$ & $\begin{array}{l}\text { Major } \\
\text { complication } \\
\text { (CD 3-5) }\end{array}$ & Median: 34.00 & Median: 33.70 & NR & $\Theta$ \\
\hline Snowden 2013 & $\begin{array}{l}\text { Mixed } \\
(389)\end{array}$ & $\mathrm{V}_{\mathrm{E}} / \mathrm{V}_{\mathrm{CO} 2}$ & $\begin{array}{l}\text { Mortality (in- } \\
\text { hospital) }\end{array}$ & $\begin{array}{l}\text { Mean (SD): } \\
35.40 \\
(6.20)\end{array}$ & $\begin{array}{l}\text { Mean }(\mathrm{SD}) \text { : } \\
36.30 \\
(4.70)\end{array}$ & $\begin{array}{c}\text { Mean difference }(95 \% \mathrm{CI}) \text { : } \\
0.90(-2.00 \text { to } 3.80)\end{array}$ & $\Theta$ \\
\hline
\end{tabular}

† Significant association between favorable preoperative cardiopulmonary exercise test (CPET) variables and absence of postoperative complications

Є No significant association between preoperative CPET variables and postoperative compilations

Significant association between unfavorable preoperative CPET variables and absence of postoperative complications

Peak $\mathrm{VO}_{2}=$ peak oxygen uptake; $\mathrm{AT}=$ anaerobic threshold; $\mathrm{V}_{\mathrm{E}} / \mathrm{V}_{\mathrm{CO} 2}=$ ventilatory equivalent for carbon dioxide

presented descriptively as the included studies presented a wide range of heterogeneity, especially using different cutoff points. Larger, prospective, cohort studies or perhaps a systematic review of individual patient data, should explore this further. Whenever possible, a subgroup analysis, involving specific groups of patients should be explored, to test whether different cutoff points for different patient cohorts provide more accurate predictive models. Future studies should attempt to use standardized CPET protocols and standardized definitions for postoperative outcomes. ${ }^{71}$ This would allow future systematic reviewers to pool data from a larger number of studies. Finally, future clinical trials should investigate the most effective exercise regime to increase preoperative physical fitness. The measurement of peak $\mathrm{VO}_{2}$ and AT before and after the preoperative exercise regime would facilitate the investigation of this effect. 


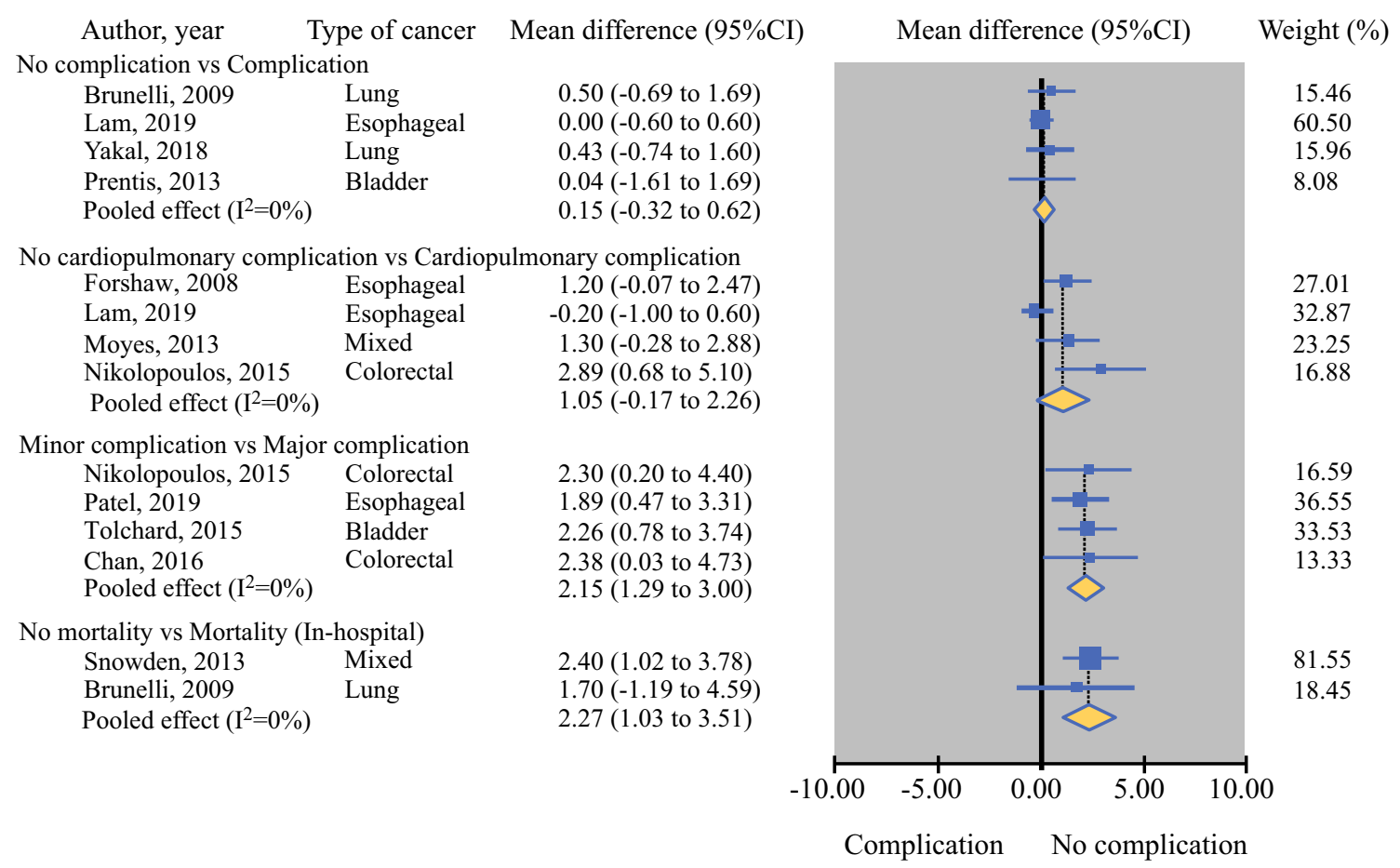

FIG. 3 Forest plot of the association between preoperative anaerobic threshold (AT) in ml $\mathrm{kg} \backslash \mathrm{min}$ and postoperative complication. Mean difference $>0$ indicate higher preoperative AT in Patients with no postoperative complications. $\mathrm{CI}=\mathrm{Confidence} \mathrm{level}$

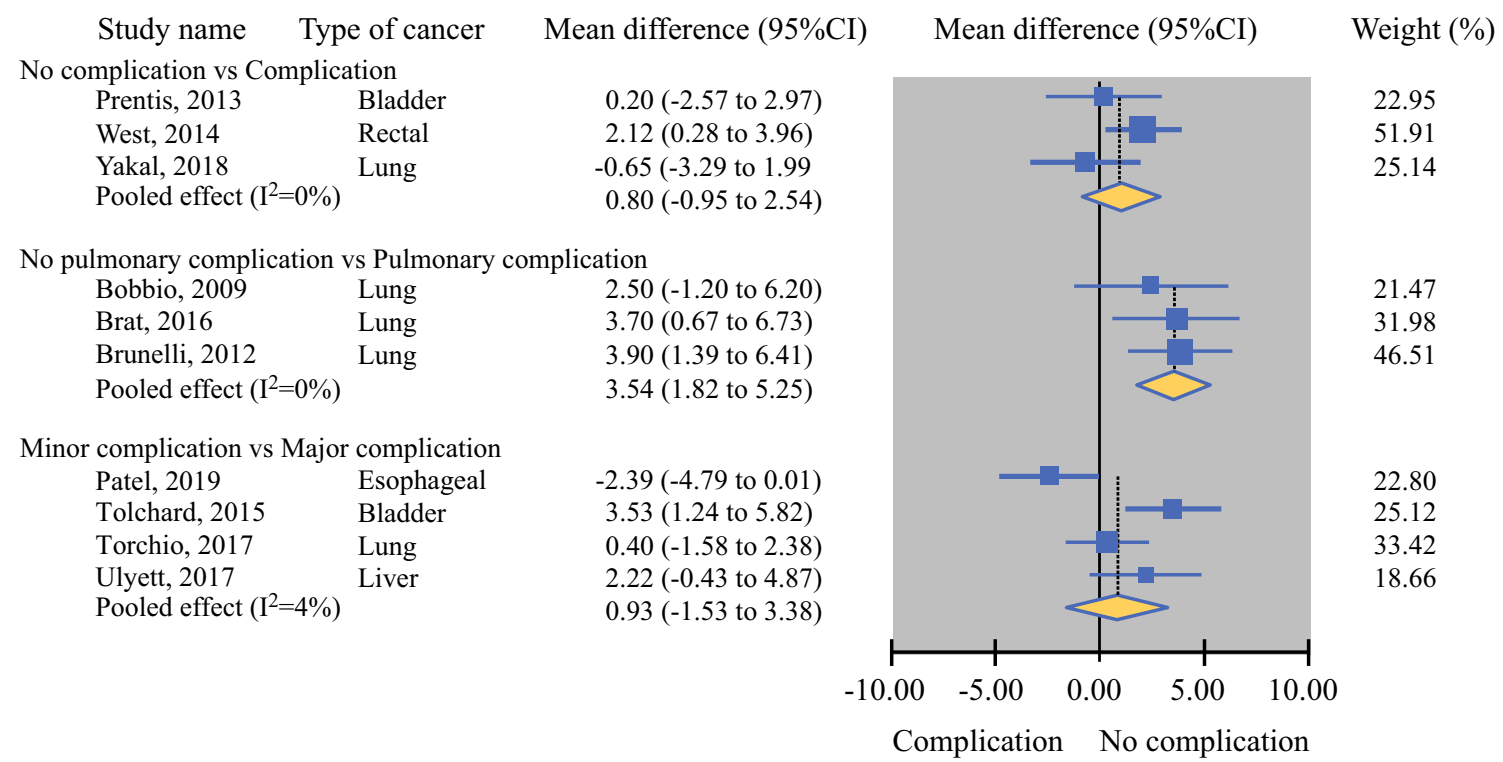

FIG. 4 Forest plot of the association between preoperative ventilatory equivalent for carbon dioxide $\left(\mathrm{V}_{\mathrm{E}} / \mathrm{VCO}_{2}\right)$ and postoperative complications. Mean difference $>0$ indicate lower preoperative $\mathrm{V}_{\mathrm{E}} / \mathrm{VCO}_{2}$ in patients with no postoperative complications. CI=Confidence level

\section{CONCLUSIONS}

This systematic review and meta-analysis has demonstrated that superior preoperative CPET values, especially peak $\mathrm{VO}_{2}$, were significantly associated with improved postoperative outcomes in patients undergoing cancer surgery. The predictive value of preoperative CPET on length of hospital stay or quality of life outcomes was not able to be determined, due to the high heterogeneity or lack of studies, respectively. Results from individual studies not included in the meta-analysis also reported positive trends. Most importantly, none of the identified studies reported a negative association between preoperative CPET values 
TABLE 5 Association between preoperative cardiopulmonary exercise test variables and length of hospital stay

\begin{tabular}{|c|c|c|c|c|c|c|c|}
\hline \multirow[t]{2}{*}{ Author year } & \multirow{2}{*}{$\begin{array}{l}\text { Cancer type } \\
(\mathrm{N})\end{array}$} & \multicolumn{2}{|c|}{ Preoperative CPET threshold } & \multicolumn{2}{|c|}{ Length of hospital stay (days) } & \multirow[t]{2}{*}{ Pooled estimates } & \multirow[t]{2}{*}{ Summary } \\
\hline & & Favorable & Unfavorable & $\begin{array}{l}\text { Favorable } \\
\text { CPET }\end{array}$ & $\begin{array}{l}\text { Unfavorable } \\
\text { CPET }\end{array}$ & & \\
\hline Bayram 2007 & Lung (55) & $\begin{array}{l}\text { Peak } \mathrm{VO}_{2}>15 \\
(\mathrm{~mL} / \mathrm{Kg} / \\
\mathrm{min})\end{array}$ & $\begin{array}{l}\text { Peak } \mathrm{VO}_{2}<15 \\
\quad(\mathrm{~mL} / \mathrm{Kg} / \\
\mathrm{min})\end{array}$ & Mean: 6 & Mean: 7 & Not reported & $\Theta$ \\
\hline Patel 2019 & $\begin{array}{l}\text { Esophageal } \\
\quad(120)\end{array}$ & $\begin{array}{l}\text { Peak } \mathrm{VO}_{2}>17 \\
(\mathrm{~mL} / \mathrm{Kg} / \\
\mathrm{min})\end{array}$ & $\begin{array}{l}\text { Peak } \mathrm{VO}_{2}<17 \\
\quad(\mathrm{~mL} / \mathrm{Kg} / \\
\mathrm{min})\end{array}$ & $\begin{array}{l}\text { Median } \\
\quad \text { (range): } 15 \\
\quad(9-153)\end{array}$ & $\begin{array}{l}\text { Median } \\
\quad \text { (range): } 16 \\
\quad(6-106)\end{array}$ & Not reported & + \\
\hline McSorley 2018 & $\begin{array}{l}\text { Colorectal } \\
\text { (38) }\end{array}$ & $\begin{array}{l}\text { Peak } \mathrm{VO}_{2}>19 \\
(\mathrm{~mL} / \mathrm{Kg} / \\
\mathrm{min})\end{array}$ & $\begin{array}{l}\text { Peak } \mathrm{VO}_{2}<19 \\
\quad(\mathrm{~mL} / \mathrm{Kg} / \\
\mathrm{min})\end{array}$ & $\begin{array}{l}\text { Median } \\
\quad \text { (range): } 9 \\
\quad(5-19)\end{array}$ & $\begin{array}{l}\text { Median } \\
\quad \text { (range): } 8 \\
\quad(3-15)\end{array}$ & Not reported & $\Theta$ \\
\hline $\begin{array}{l}\text { Kasivisvanathan } \\
2015\end{array}$ & Liver (104) & \multicolumn{2}{|c|}{ Peak $\mathrm{VO}_{2}(\mathrm{~mL} / \mathrm{Kg} / \mathrm{min})$} & Not reported & Not reported & $\begin{array}{l}\text { Hazard ratio }(95 \% \mathrm{CI}): \\
1.15(0.99-1.40)\end{array}$ & $\Theta$ \\
\hline Dunne 2014 & Liver (197) & \multicolumn{2}{|c|}{ Peak $\mathrm{VO}_{2}(\mathrm{~mL} / \mathrm{Kg} / \mathrm{min})$} & Not reported & Not reported & $\begin{array}{l}\text { Hazard ratio }(95 \% \mathrm{CI}): \\
1.10(0.98-1.04)\end{array}$ & $\Theta$ \\
\hline Sinclair 2017 & $\begin{array}{l}\text { Esophageal } \\
\text { (240) }\end{array}$ & \multicolumn{2}{|c|}{ Peak $\mathrm{VO}_{2}(\mathrm{~mL} / \mathrm{Kg} / \mathrm{min})$} & Not reported & Not reported & $\begin{array}{l}\text { Odds ratio }(95 \% \mathrm{CI}): 1.00 \\
\quad(1.0-1.1)\end{array}$ & ( \\
\hline Chan 2016 & $\begin{array}{l}\text { Colorectal } \\
\quad(48)\end{array}$ & \multicolumn{2}{|c|}{ Peak $\mathrm{VO}_{2}(\mathrm{~mL} / \mathrm{Kg} / \mathrm{min})$} & Not reported & Not reported & Not reported & $\Theta$ \\
\hline $\begin{array}{l}\text { Chandrabalan } \\
2013\end{array}$ & $\begin{array}{l}\text { Pancreas } \\
\quad(93)\end{array}$ & $\begin{array}{c}\mathrm{AT} \geq 10(\mathrm{~mL} / \\
\mathrm{Kg} / \mathrm{min})\end{array}$ & $\begin{array}{c}\mathrm{AT}<10(\mathrm{~mL} / \\
\mathrm{Kg} / \mathrm{min})\end{array}$ & Not reported & Not reported & $\begin{array}{l}\text { Hazard ratio }(95 \% \mathrm{CI}) \text { : } \\
1.70(1.1-2.6)\end{array}$ & + \\
\hline Ausania 2012 & $\begin{array}{l}\text { Pancreas } \\
\text { (124) }\end{array}$ & $\begin{array}{c}\mathrm{AT} \geq 10.1 \\
(\mathrm{~mL} / \mathrm{Kg} / \\
\mathrm{min})\end{array}$ & $\begin{array}{c}\mathrm{AT}<10.1 \\
\quad(\mathrm{~mL} / \mathrm{Kg} / \\
\mathrm{min})\end{array}$ & $\begin{array}{l}\text { Median } \\
\quad \text { (range): } \\
17.5(8-99)\end{array}$ & $\begin{array}{l}\text { Median } \\
\quad \text { (range): } 29.4 \\
\quad(12-54)\end{array}$ & Not reported & \\
\hline Patel 2019 & $\begin{array}{l}\text { Esophageal } \\
\quad(120)\end{array}$ & $\begin{array}{c}\mathrm{AT} \geq 10.5 \\
(\mathrm{~mL} / \mathrm{Kg} / \\
\mathrm{min})\end{array}$ & $\begin{array}{c}\mathrm{AT}<10.5 \\
\quad(\mathrm{~mL} / \mathrm{Kg} / \\
\mathrm{min})\end{array}$ & Median: 16 & Median: 16 & Not reported & $\Theta$ \\
\hline Wilson 2010 & $\begin{array}{l}\text { Mixed } \\
(847)\end{array}$ & $\begin{array}{c}\mathrm{AT} \geq 10.9 \\
(\mathrm{~mL} / \mathrm{Kg} / \\
\mathrm{min})\end{array}$ & $\begin{array}{c}\mathrm{AT}<10.9 \\
\quad(\mathrm{~mL} / \mathrm{Kg} / \\
\mathrm{min})\end{array}$ & Median: 8 & Median: 9 & Not reported & $\oplus$ \\
\hline Lamb 2016 & $\begin{array}{r}\text { Bladder } \\
(111)\end{array}$ & $\begin{array}{c}\mathrm{AT} \geq 11(\mathrm{~mL} / \\
\mathrm{Kg} / \mathrm{min})\end{array}$ & $\begin{array}{c}\mathrm{AT}<11(\mathrm{~mL} / \\
\mathrm{Kg} / \mathrm{min})\end{array}$ & $\begin{array}{l}\text { Median (IQR): } \\
10(7-13)\end{array}$ & $\begin{array}{l}\text { Median (IQR): } \\
11 \\
\quad(7.5-14.5)\end{array}$ & Not reported & $\Theta$ \\
\hline $\begin{array}{l}\text { Chandrabalan } \\
2013\end{array}$ & $\begin{array}{l}\text { Pancreas } \\
\quad(93)\end{array}$ & $\begin{array}{c}\mathrm{AT} \geq 11(\mathrm{~mL} / \\
\mathrm{Kg} / \mathrm{min})\end{array}$ & $\begin{array}{c}\mathrm{AT}<11(\mathrm{~mL} / \\
\mathrm{Kg} / \mathrm{min})\end{array}$ & Not reported & Not reported & $\begin{array}{l}\text { Hazard ratio }(95 \% \mathrm{CI}): \\
1.40(0.90-2.20)\end{array}$ & $\Theta$ \\
\hline Forshaw 2008 & $\begin{array}{l}\text { Esophageal } \\
\quad(75)\end{array}$ & $\begin{array}{c}\mathrm{AT}>11(\mathrm{~mL} / \\
\mathrm{Kg} / \mathrm{min})\end{array}$ & $\begin{array}{c}\mathrm{AT}<11(\mathrm{~mL} / \\
\mathrm{Kg} / \mathrm{min})\end{array}$ & $\begin{array}{l}\text { Mean (SD): } 19 \\
\quad(23)\end{array}$ & $\begin{array}{l}\text { Mean (SD): } 19 \\
\quad(9)\end{array}$ & $\begin{array}{c}\text { Mean difference }(95 \% \mathrm{CI}) \text { : } \\
0.00(-13.30 \text { to } 13.30)\end{array}$ & $\Theta$ \\
\hline Lamb 2016 & $\begin{array}{r}\text { Bladder } \\
(111)\end{array}$ & $\begin{array}{c}\mathrm{AT} \geq 12(\mathrm{~mL} / \\
\mathrm{Kg} / \mathrm{min})\end{array}$ & $\begin{array}{c}\mathrm{AT}<12(\mathrm{~mL} / \\
\mathrm{Kg} / \mathrm{min})\end{array}$ & $\begin{array}{l}\text { Median (IQR): } \\
\quad 9(8-12)\end{array}$ & $\begin{array}{l}\text { Median (IQR): } \\
\quad 11(8-15)\end{array}$ & Not reported & $\Theta$ \\
\hline Chan 2016 & $\begin{array}{l}\text { Colorectal } \\
\quad(48)\end{array}$ & $\mathrm{AT}(\mathrm{mL} / \mathrm{Kg} / \mathrm{mi}$ & & Not reported & Not reported & Not reported & $E$ \\
\hline
\end{tabular}


TABLE 5 continued

\begin{tabular}{|c|c|c|c|c|c|c|c|}
\hline \multirow[t]{2}{*}{ Author year } & \multirow{2}{*}{$\begin{array}{l}\text { Cancer type } \\
(\mathrm{N})\end{array}$} & \multicolumn{2}{|c|}{ Preoperative CPET threshold } & \multicolumn{2}{|c|}{ Length of hospital stay (days) } & \multirow[t]{2}{*}{ Pooled estimates } & \multirow[t]{2}{*}{ Summary } \\
\hline & & Favorable & Unfavorable & $\begin{array}{l}\text { Favorable } \\
\text { CPET }\end{array}$ & $\begin{array}{l}\text { Unfavorable } \\
\text { CPET }\end{array}$ & & \\
\hline Sinclair 2017 & $\begin{array}{l}\text { Esophageal } \\
\quad(240)\end{array}$ & $\mathrm{AT}(\mathrm{mL} / \mathrm{Kg} / \mathrm{min})$ & & Not reported & Not reported & $\begin{array}{l}\text { Odds ratio }(95 \% \text { CI): } 1.00 \\
\quad(1.00-1.10)\end{array}$ & \\
\hline Mann 2020 & $\begin{array}{c}\text { Colorectal } \\
\text { (1193) }\end{array}$ & $\mathrm{V}_{\mathrm{E}} / \mathrm{V}_{\mathrm{CO} 2} \leq 34$ & $\mathrm{~V}_{\mathrm{E}} / \mathrm{V}_{\mathrm{CO} 2}>34$ & Not reported & Not reported & $\begin{array}{l}\text { Odds ratio }(95 \% \mathrm{CI}): 1.70 \\
\quad(1.30-2.20)\end{array}$ & \\
\hline Sinclair 2017 & $\begin{array}{l}\text { Esophageal } \\
\quad(240)\end{array}$ & $\mathrm{V}_{\mathrm{E}} / \mathrm{V}_{\mathrm{CO} 2}$ & & Not reported & Not reported & $\begin{array}{l}\text { Odds ratio }(95 \% \text { CI): } 0.90 \\
\quad(0.90-0.90)\end{array}$ & \\
\hline Dunne 2014 & Liver (197) & $\mathrm{V}_{\mathrm{E}} / \mathrm{V}_{\mathrm{CO} 2}$ & & Not reported & Not reported & $\begin{array}{c}\text { Hazard ratio }(95 \% \mathrm{CI}): \\
0.90(0.90-1.00)\end{array}$ & $=$ \\
\hline
\end{tabular}

Significant association between favorable preoperative cardiopulmonary exercise test (CPET) variables and length of hospital stay

No significant association between preoperative CPET variables and length of hospital stay

Significant association between unfavorable preoperative CPET variables and length of hospital stay

Peak $\mathrm{VO}_{2}=$ peak oxygen uptake; $\mathrm{AT}=$ anaerobic threshold; $\mathrm{V}_{\mathrm{E}} / \mathrm{V}_{\mathrm{CO} 2}=$ ventilatory equivalent for carbon dioxide

and postoperative outcomes. The authors of this review recommend the use of preoperative CPET before cancer surgery to predict postoperative outcomes.

DISCLOSURE The authors declare no conflict of interest.

\section{REFERENCES}

1. Bray F, Ferlay J, Soerjomataram I, Siegel RL, Torre LA, Jemal A. Global cancer statistics 2018: GLOBOCAN estimates of incidence and mortality worldwide for 36 cancers in 185 countries. CA Cancer J Clin. 2018;68(6):394-424.

2. Steffens D, Koh C, Ansari N, Solomon MJ, Brown K, McBride $\mathrm{K}$, et al. Quality of life after cytoreductive surgery and hyperthermic intraperitoneal chemotherapy: early results from a prospective cohort study of 115 patients. Ann Surg Oncol. 2020;27(10):3986-94.

3. Steffens D, Solomon MJ, Young JM, Koh C, Venchiarutti RL, Lee $\mathrm{P}$, et al. Cohort study of long-term survival and quality of life following pelvic exenteration. BJS Open. 2018;2(5):328-35.

4. Bolliger CT, Perruchoud AP. Functional evaluation of the lung resection candidate. Eur Respir J. 1998;11(1):198-212.

5. Sivakumar J, Sivakumar H, Read M, Sinclair RCF, Snowden CP, Hii MW. The role of cardiopulmonary exercise testing as a risk assessment tool in patients undergoing oesophagectomy: a systematic review and meta-analysis. Ann Surg Oncol. 2020;27(10):3783-96.

6. Levett DZH, Jack S, Swart M, Carlisle J, Wilson J, Snowden C, et al. Perioperative cardiopulmonary exercise testing (CPET): consensus clinical guidelines on indications, organization, conduct, and physiological interpretation. $B r \quad J$ Anaesth. 2018;120(3):484-500.
7. Benzo R, Kelley GA, Recchi L, Hofman A, Sciurba F. Complications of lung resection and exercise capacity: a meta-analysis. Respir Med. 2007;101(8):1790-7.

8. Lee CHA, Kong JC, Ismail H, Riedel B, Heriot A. Systematic review and meta-analysis of objective assessment of physical fitness in patients undergoing colorectal cancer surgery. Dis Colon Rectum. 2018;61(3):400-9.

9. Moran J, Wilson F, Guinan E, McCormick P, Hussey J, Moriarty $\mathrm{J}$. Role of cardiopulmonary exercise testing as a risk-assessment method in patients undergoing intra-abdominal surgery: a systematic review. Br J Anaesth. 2016;116(2):177-91.

10. Lam S, Hart A. Cardiopulmonary Exercise Testing for Predicting Early Outcomes after Major Cancer Resection: A Systematic Review. J Anesth Periop Med. 2018;5:136-48.

11. Stubbs DJ, Grimes LA, Ercole A. Performance of cardiopulmonary exercise testing for the prediction of post-operative complications in noncardiopulmonary surgery: a systematic review. PLoS One. 2020;15(2): 0226480.

12. Wijeysundera DN, Pearse RM, Shulman MA, Abbott TEF, Torres E, Ambosta A, et al. Assessment of functional capacity before major non-cardiac surgery: an international, prospective cohort study. Lancet (London, England). 2018;391(10140):2631-40.

13. Stroup DF, Berlin JA, Morton SC, Olkin I, Williamson GD, Rennie D, et al. Meta-analysis of observational studies in epidemiology: a proposal for reporting Meta-analysis Of Observational Studies in Epidemiology (MOOSE) group. JAMA. 2000;283(15):2008-12.

14. Hayden JA, van der Windt DA, Cartwright JL, Côté P, Bombardier C. Assessing bias in studies of prognostic factors. Ann Intern Med. 2013;158(4):280-6.

15. Hozo SP, Djulbegovic B, Hozo I. Estimating the mean and variance from the median, range, and the size of a sample. $B M C$ Med Res Method. 2005;5(1):13.

16. Lamb BW, Tan WS, Eneje P, Bruce D, Jones A, Ahmad I, et al. Benefits of robotic cystectomy with intracorporeal diversion for 
patients with low cardiorespiratory fitness: a prospective cohort study. Urol Oncol Sem Original Invest. 2016;34(9):417.e17-23.

17. Prentis JM, Trenell MI, Vasdev N, French R, Dines G, Thorpe A, et al. Impaired cardiopulmonary reserve in an elderly population is related to postoperative morbidity and length of hospital stay after radical cystectomy. BJU Int. 2013;112(2):E13-19.

18. Tolchard S, Angell J, Pyke M, Lewis S, Dodds N, Darweish A, et al. Cardiopulmonary reserve as determined by cardiopulmonary exercise testing correlates with length of stay and predicts complications after radical cystectomy. BJU Int. 2015;115(4):554-61.

19. Bowles TA, Sanders KM, Colson M, Watters DA. Simplified risk stratification in elective colorectal surgery. ANZ $J$ Surg. 2008;78(1-2):24-7.

20. Chan KE, Pathak S, Smart NJ, Batchelor N, Daniels IR. The impact of cardiopulmonary exercise testing on patients over the age of 80 undergoing elective colorectal cancer surgery. Colorectal Dis. 2016;18(6):578-85.

21. Mann J, Williams M, Wilson J, Yates D, Harrison A, Doherty P, et al. Exercise-induced myocardial dysfunction detected by cardiopulmonary exercise testing is associated with increased risk of mortality in major oncological colorectal surgery. $\mathrm{Br} J$ Anaesth. 2020;124(4):473-9.

22. McSorley ST, Roxburgh CSD, Horgan PG, McMillan DC. The relationship between cardiopulmonary exercise test variables, the systemic inflammatory response, and complications following surgery for colorectal cancer. Periop Med. 2018;7(1):1-7.

23. Nikolopoulos I, Ellwood M, George M, Carapeti E, Williams A. Cardiopulmonary exercise testing versus spirometry as predictors of cardiopulmonary complications after colorectal surgery. Eur Surg Acta Chirurg Austriaca. 2015;47(6):324-30.

24. Forshaw MJ, Strauss DC, Davies AR, Wilson D, Lams B, Pearce A, et al. Is cardiopulmonary exercise testing a useful test before esophagectomy? Ann Thorac Surg. 2008;85(1):294-9.

25. Lam S, Alexandre L, Hardwick G, Hart AR. The association between preoperative cardiopulmonary exercise-test variables and short-term morbidity after esophagectomy: a hospital-based cohort study. Surgery (United States). 2019;166(1):28-33.

26. Nagamatsu Y, Shima I, Yamana H, Fujita H, Shirouzu K, Ishitake T. Preoperative evaluation of cardiopulmonary reserve with the use of expired gas analysis during exercise testing in patients with squamous cell carcinoma of the thoracic esophagus. $J$ Thorac Cardiovasc Surg. 2001;121(6):1064-8.

27. Nagamatsu Y, Yamana H, Fujita H, Hiraki H, Matsuo T, Mitsuoka $\mathrm{M}$, et al. The simultaneous evaluation of preoperative cardiopulmonary functions of esophageal cancer patients in the analysis of expired gas with exercise testing. Nippon Kyobu Geka Gakkai Zasshi. 1994;42(11):2037-40.

28. Patel N, Powell AG, Wheat JR, Brown C, Appadurai IR, Davies $\mathrm{RG}$, et al. Cardiopulmonary fitness predicts postoperative major morbidity after esophagectomy for patients with cancer. Physiol Rep. 2019;7(14):e14174.

29. Sinclair RCF, Phillips AW, Navidi M, Griffin SM, Snowden CP. Pre-operative variables including fitness associated with complications after oesophagectomy. Anaesthesia. 2017;72(12):1501-7.

30. Dunne DFJ, Jones RP, Lythgoe DT, Pilkington FJ, Palmer DH, Malik HZ, et al. Cardiopulmonary exercise testing before liver surgery. J Surg Oncol. 2014;110(4):439-44.

31. Kasivisvanathan R, Abbassi-Ghadi N, McLeod ADM, Oliver A, Baikady RR, Jhanji S, et al. Cardiopulmonary exercise testing for predicting postoperative morbidity in patients undergoing hepatic resection surgery. HPB. 2015;17(7):637-43.

32. Ulyett S, Shahtahmassebi G, Aroori S, Bowles MJ, Briggs CD, Wiggans MG, et al. Comparison of risk-scoring systems in the prediction of outcome after liver resection. Periop Med. 2017;6(1):1-7.

33. Bayram AS, Candan T, Gebitekin C. Preoperative maximal exercise oxygen consumption test predicts postoperative pulmonary morbidity following major lung resection. Respirology. 2007;12(4):505-10.

34. Bechard D, Wetstein L. Assessment of exercise oxygen consumption as preoperative criterion for lung resection. Ann Thorac Surg. 1987;44(4):344-9.

35. Bobbio A, Chetta A, Internullo E, Ampollini L, Carbognani P, Bettati S, et al. Exercise capacity assessment in patients undergoing lung resection. Eur $J$ Cardiothorac Surg. 2009;35(3):419-22.

36. Bolliger CT, Wyser C, Roser H, Soler M, Perruchoud AP. Lung scanning and exercise testing for the prediction of postoperative performance in lung resection candidates at increased risk for complications. Chest. 1995;108(2):341-8.

37. Brat K, Tothova Z, Merta Z, Taskova A, Homolka P, Vasakova M, et al. Resting End-Tidal Carbon Dioxide Predicts Respiratory Complications in Patients Undergoing Thoracic Surgical Procedures. Ann Thorac Surg. 2016;102(5):1725-30.

38. Brunelli A, Belardinelli R, Pompili C, Xiumé F, Refai M, Salati $\mathrm{M}$, et al. Minute ventilation-to-carbon dioxide output (VE/VCO2) slope is the strongest predictor of respiratory complications and death after pulmonary resection. Ann Thorac Surg. 2012;93(6):1802-6.

39. Brunelli A, Belardinelli R, Refai M, Salati M, Socci L, Pompili $\mathrm{C}$, et al. Peak oxygen consumption during cardiopulmonary exercise test improves risk stratification in candidates to major lung resection. Chest. 2009;135(5):1260-7.

40. Brutsche MH, Spiliopoulos A, Bolliger CT, Licker M, Frey JG, Tschopp JM. Exercise capacity and extent of resection as predictors of surgical risk in lung cancer. Eur Resp J. 2000;15(5):828-32.

41. Dales RE, Dionne G, Leech JA, Lunau M, Schweitzer I. Preoperative prediction of pulmonary complications following thoracic surgery. Chest. 1993;104(1):155-9.

42. Epstein SK, Faling LJ, Daly BD, Celli BR. Predicting complications after pulmonary resection Preoperative exercise testing vs a multifactorial cardiopulmonary risk index. Chest. 1993;104(3):694-700.

43. Fang Y, Ma G, Lou N, Liao W, Wang D. Preoperative maximal oxygen uptake and exercise-induced changes in pulse oximetry predict early postoperative respiratory complications in lung cancer patients. Scand J Surg. 2014;103(3):201-8.

44. Han J, Lee G. Preoperative assessment of risk factors as a predictor of 30-day mortality and morbidity after lung resection for lung cancer. EWHA Med J. 2007;30(1):37-46.

45. Kasikcioglu E, Toker A, Tanju S, Arzuman P, Kayserilioglu A, Dilege $\mathrm{S}$, et al. Oxygen uptake kinetics during cardiopulmonary exercise testing and postoperative complications in patients with lung cancer. Lung Cancer. 2009;66(1):85-8.

46. Licker M, Schnyder JM, Frey JG, Diaper J, Cartier V, Inan C, et al. Impact of aerobic exercise capacity and procedure-related factors in lung cancer surgery. Eur Respir J. 2011;37(5):1189-98.

47. Loewen GM, Watson D, Kohman L, Herndon JE 2nd, Shennib H, Kernstine K, et al. Preoperative exercise Vo2 measurement for lung resection candidates: results of Cancer and Leukemia Group B Protocol 9238. J Thorac Oncol. 2007;2(7):619-25.

48. Mao YS, He J, Yan SP, Dong JS, Cheng GY, Sun KL, et al. Cardiopulmonary exercise testing in the evaluation of high risk patients with lung cancer. Chinese Med J. 2010;123(21):3089-94.

49. Markos J, Mullan BP, Hillman DR, Musk AW, Antico VF, Lovegrove FT, et al. Preoperative assessment as a predictor of mortality and morbidity after lung resection. Am Rev Respir Dis. 1989;139(4):902-10. 
50. Miyazaki T, Callister MEJ, Franks K, Dinesh P, Nagayasu T, Brunelli A. Minute ventilation-to-carbon dioxide slope is associated with postoperative survival after anatomical lung resection. Lung Cancer. 2018;125:218-22.

51. Morice RC, Peters EJ, Ryan MB, Putnam JB, Ali MK, Roth JA. Exercise testing in the evaluation of patients at high risk for complications from lung resection. Chest. 1992;101(2):356-61.

52. Nagamatsu Y, Shima I, Hayashi A, Yamana H, Shirouzu K, Ishitake T. Preoperative spirometry versus expired gas analysis during exercise testing as predictors of cardiopulmonary complications after lung resection. Surg. 2004;34(2):107-10.

53. Nagamatsu Y, Terazaki Y, Muta F, Yamana H, Shirouzu K, Ishitake T. Expired gas analysis during exercise testing prepneumonectomy. Surg Today. 2005;35(12):1021-5.

54. Pate P, Tenholder MF, Griffin JP, Eastridge CE, Weiman DS. Preoperative assessment of the high-risk patient for lung resection. Ann Thorac Surg. 1996;61(5):1494-500.

55. Rodrigues F, Grafino M, Faria I, da Mata JP, Papoila AL, Felix F. Surgical risk evaluation of lung cancer in COPD patients: a cohort observational study. Revista Portuguesa Pneumol (English Edition). 2016;22(5):266-72.

56. Torchio R, Mazzucco A, Guglielmo M, Giardino R, Ciacco C, Ardissone F. Minute ventilation to carbon dioxide output (V'E/ V'CO2 slope) is the strongest death predictor before larger lung resections. Monaldi Arch Chest Dis. 2017;87(3):817.

57. Villani F, Busia A. Preoperative evaluation of patients submitted to pneumonectomy for lung carcinoma: role of exercise testing. Tumori. 2004;90(4):405-9.

58. Win T, Jackson A, Sharples L, Groves AM, Wells FC, Ritchie AJ, et al. Cardiopulmonary exercise tests and lung cancer surgical outcome. Chest. 2005;127(4):1159-65.

59. Yakal S, Sofyali S, Ozkan B, Yildiz S, Toker A, Kasikcioglu E. Oxygen uptake efficiency slope and prediction of post-operative morbidity and mortality in patients with lung cancer. Lung. 2018;196(2):255-62.

60. Begum SS, Papagiannopoulos K, Falcoz PE, Decaluwe H, Salati $\mathrm{M}$, Brunelli A. Outcome after video-assisted thoracoscopic surgery and open pulmonary lobectomy in patients with low $\mathrm{VO}_{2}$ max: a case-matched analysis from the ESTS database $\dagger$. Eur $J$ Cardiothorac Surg. 2016;49(4):1054-8.

61. Ausania F, Snowden CP, Prentis JM, Holmes LR, Jaques BC, White SA, et al. Effects of low cardiopulmonary reserve on pancreatic leak following pancreaticoduodenectomy. Br J Surg. 2012;99(9):1290-4.

62. Chandrabalan VV, McMillan DC, Carter R, Kinsella J, McKay CJ, Carter CR, et al. Pre-operative cardiopulmonary exercise testing predicts adverse post-operative events and non-progression to adjuvant therapy after major pancreatic surgery. $H P B$. 2013;15(11):899-907.

63. Junejo MA, Mason JM, Sheen AJ, Bryan A, Moore J, Foster P, et al. Cardiopulmonary exercise testing for preoperative risk assessment before pancreaticoduodenectomy for cancer. Ann Surg Oncol. 2014;21(6):1929-36.

64. West MA, Parry MG, Lythgoe D, Barben CP, Kemp GJ, Grocott $\mathrm{MP}$, et al. Cardiopulmonary exercise testing for the prediction of morbidity risk after rectal cancer surgery. $\mathrm{Br} \quad J$ Surg. 2014;101(9):1166-72.

65. Huang GH, Ismail H, Murnane A, Kim P, Riedel B. Structured exercise program prior to major cancer surgery improves cardiopulmonary fitness: a retrospective cohort study. Support Care Cancer. 2016;24(5):2277-85.

66. Moyes LH, McCaffer CJ, Carter RC, Fullarton GM, Mackay CK, Forshaw MJ. Cardiopulmonary exercise testing as a predictor of complications in oesophagogastric cancer surgery. Ann $R$ Coll Surg Engl. 2013;95(2):125-30.

67. Drummond RJ, Vass D, Wadhawan H, Craig CF, MacKay CK, Fullarton GM, et al. Routine pre- and post-neoadjuvant chemotherapy fitness testing is not indicated for oesophagogastric cancer surgery. Ann R Coll Surg Engl. 2018;100(7):515-9.

68. Snowden CP, Prentis J, Jacques B, Anderson H, Manas D, Jones $\mathrm{D}$, et al. Cardiorespiratory fitness predicts mortality and hospital length of stay after major elective surgery in older people. Ann Surg. 2013;257(6):999-1004.

69. Whibley J, Peters CJ, Halliday LJ, Chaudry AM, Allum WH. Poor performance in incremental shuttle walk and cardiopulmonary exercise testing predicts poor overall survival for patients undergoing esophago-gastric resection. Eur J Surg Oncol. 2018;44(5):594-9.

70. Wilson RJ, Davies S, Yates D, Redman J, Stone M. Impaired functional capacity is associated with all-cause mortality after major elective intra-abdominal surgery. $\mathrm{Br} J$ Anaesth. 2010;105(3):297-303.

71. Moonesinghe SR, Jackson AIR, Boney O, Stevenson N, Chan MTV, Cook TM, et al. Systematic review and consensus definitions for the standardised endpoints in perioperative medicine initiative: patient-centred outcomes. $\mathrm{Br} J$ Anaesth. 2019;123(5):664-70.

Publisher's Note Springer Nature remains neutral with regard to jurisdictional claims in published maps and institutional affiliations. 